\title{
Multicriteria Decision-Making Method and Application in the Setting of Trapezoidal Neutrosophic Z-Numbers
}

\author{
Rui Yong, ${ }^{1}$ Jun Ye $\mathbb{D}^{1,2}$ and Shigui ${ }^{1 u^{1}}$ \\ ${ }^{1}$ School of Civil and Environmental Engineering, Ningbo University, Ningbo 315211, China \\ ${ }^{2}$ School of Mechanical and Electrical Engineering, Shaoxing University, Shaoxing 312000, China \\ Correspondence should be addressed to Jun Ye; yejun1@nbu.edu.cn
}

Received 10 November 2020; Revised 30 November 2020; Accepted 27 January 2021; Published 5 March 2021

Academic Editor: Basil K.Papadopoulos

Copyright (c) 2021 Rui Yong et al. This is an open access article distributed under the Creative Commons Attribution License, which permits unrestricted use, distribution, and reproduction in any medium, provided the original work is properly cited.

The information expression and modeling of decision-making are critical problems in the fuzzy decision theory and method. However, existing trapezoidal neutrosophic numbers (TrNNs) and neutrosophic Z-numbers (NZNs) and their multicriteria decision-making (MDM) methods reveal their insufficiencies, such as without considering the reliability measures in TrNN and continuous Z-numbers in NZN. To overcome the insufficiencies, it is necessary that one needs to propose trapezoidal neutrosophic Z-numbers (TrNZNs), their aggregation operations, and an MDM method for solving MDM problems with TrNZN information. Hence, this study first proposes a TrNZN set, some basic operations of TrNZNs, and the score and accuracy functions of TrNZN and their ranking laws. Then, the TrNZN weighted arithmetic averaging (TrNZNWAA) and TrNZN weighted geometric averaging (TrNZNWGA) operators are presented based on the operations of TrNZNs. Next, an MDM approach using the proposed aggregation operators and score and accuracy functions is established to carry out MDM problems under the environment of TrNZNs. In the end, the established MDM approach is applied to an MDM example of software selection for revealing its rationality and efficiency in the setting of TrNZNs. The main advantage of this study is that the established approach not only makes assessment information continuous and reliable but also strengthens the decision rationality and efficiency in the setting of TrNZNs.

\section{Introduction}

In fuzzy decision-making problems, various new fuzzy decision-making methods [1-3] have received many applications under neutrosophic, simplified neutrosophic hesitant fuzzy, and bipolar neutrosophic environments. Then, triangular and trapezoidal fuzzy numbers are usually used for real decision-making problems because they can be depicted by the continuous fuzzy numbers of membership functions rather than exact/discrete fuzzy values. Hence, some researchers extended triangular fuzzy numbers to intuitionistic fuzzy sets (IFSs) and presented triangular intuitionistic fuzzy sets (TIFSs), where the values of the membership and nonmembership functions are triangular fuzzy numbers, and some triangular intuitionistic fuzzy aggregation operators for multicriteria decision-making (MDM) problems with triangular intuitionistic fuzzy information [4-7]. As the extension of TIFSs, Ye [8] introduced a trapezoidal intuitionistic fuzzy set (TrIFS), in which the values of its membership and nonmembership functions are trapezoidal fuzzy numbers rather than triangular fuzzy numbers, and some prioritized weighted aggregation operators of trapezoidal intuitionistic fuzzy numbers (TrIFNs) for MDM problems with TrIFNs. However, TIFSs and TrIFSs cannot depict inconsistence and indeterminacy information. Hence, Ye [9] generalized TrIFS and proposed a trapezoidal neutrosophic set (TrNS), in which the values of its truth, falsity, and indeterminacy membership functions are trapezoidal fuzzy numbers, to express incomplete, indeterminate, and inconsistent information, and then he presented some basic operations of trapezoidal neutrosophic numbers (TrNNs), score and accuracy functions of TrNNs, and TrNN weighted arithmetic averaging (TrNNWAA) and $\operatorname{TrNN}$ weighted geometric 
averaging (TrNNWGA) operators for MDM problems in the setting of TrNNs. Then, some researchers utilized the integrated approach [10] and defuzzification method [11] for the evaluation and MDM problems with interval-valued TrNNs. Further, Giri et al. [12] applied TOPSIS method in MDM problems with interval-valued TrNNs. Also, Jana et al. [13] and Khatter [14] presented some basic operations of interval-valued TrNNs, score and accuracy functions of an interval-valued TrNN, and the interval-valued TrNNWAA and TrNNWGA operators for MDM problems in the setting of interval-valued TrNNs.

The notion of a Z-number introduced by Zadeh [15] is described by a fuzzy number and its reliability measure to strengthen the reliability of the fuzzy information. After that, $\mathrm{Z}$-numbers have been used for many areas [16-22]. Based on the truth, falsity, and indeterminacy Z-numbers, Du et al. [23] extended the Z-number concept and proposed neutrosophic Z-numbers (NZNs) to enhance the reliability of the neutrosophic information, and then they presented basic operations of NZNs, score and accuracy functions of NZN, and the NZN weighted geometric averaging (NZNWGA) and NZN weighted arithmetic averaging (NZNWAA) operators and further established their MDM method under the environment of NZNs.

However, TrNN is described only by the trapezoidal fuzzy numbers of its truth, falsity, and indeterminacy membership functions without considering their reliability measures, while NZN is depicted only by exact/discrete truth, falsity, and indeterminacy Z-numbers rather than continuous Z-numbers. Hence, TrNN and NZN and their MDM methods reveal their insufficiencies in their information expressions and applications. To express both the continuous Z-numbers of truth, falsity, and indeterminacy membership functions and the reliability measures in MDM problems, it is necessary that this study needs to propose an MDM method based on trapezoidal neutrosophic Z-numbers (TrNZNs) to make up such insufficiencies of existing information expressions and MDM methods in the environments of TrNNs and NZNs. To do so, the main aims of this article are (1) to propose a TrNZN set and some basic operations of TrNZNs, (2) to introduce score and accuracy functions of TrNZN for ranking TrNZNs, (3) to put forward the TrNZNWAA and TrNZNWGA operators for aggregating TrNZNs, (4) to develop a MDM approach using the proposed aggregation operators and score and accuracy functions for solving MDM problems under the environment of TrNZNs, and (5) to apply the established MDM approach to an MDM example of software selection for revealing its efficiency in the setting of TrNZNs.

The rest of the article is composed of the following sections. Section 2 introduces some basic notions of TrNNs as preliminaries of this study. Section 3 proposes a TrNZN set, basic operations of TrNZNs, the score and accuracy functions of $\operatorname{TrNZN}$, and their ranking laws of TrNZNs. Then, the TrNZNWAA and TrNZNWGA operators and their relative properties are presented in section 4 . Section 5 develops an MDM approach using the TrNZNWAA and TrNZNWGA operators and score and accuracy functions of TrNZNs. In Section 6, the developed MDM approach is applied to an MDM example of software selection to indicate its efficiency in the setting of TrNZNs. In the end, conclusions and further study are contained in Section 7.

\section{Preliminaries of TrNSs}

In this section, we introduce preliminaries of TrNSs, including TrNNs, operations of TrNNs, two TrNN weighted aggregation operators, and score and accuracy functions of TrNNs for ranking TrNNs.

Ye [9] first proposed TrNS in a universe set $U$, which is denoted as

$$
\widetilde{Y}=\left\{\left\langle u, \mathrm{TN}_{\tilde{Y}}(u), \operatorname{IN}_{\tilde{Y}}(u), \mathrm{FN}_{\widetilde{Y}}(u)\right\rangle, \quad u \in U\right\},
$$

where $\operatorname{TN}_{\widetilde{Y}}(u) \subseteq[0,1], \operatorname{IN}_{\widetilde{Y}}(u) \subseteq[0,1]$, and $\operatorname{FN}_{\widetilde{Y}}(u) \subseteq[0,1]$ are the truth, indeterminacy, and falsity membership functions; then their values are three trapezoidal fuzzy numbers $\mathrm{TN}_{\tilde{Y}}(u)=\left(\mathrm{TN}_{1}(u), \mathrm{TN}_{2}(u), \mathrm{TN}_{3}(u), \mathrm{TN}_{4}(u)\right): \mathrm{U} \longrightarrow[0$, 1], $\operatorname{IN}_{\tilde{Y}}(u)=\left(\operatorname{IN}_{1}(u), \operatorname{IN}_{2}(u), \operatorname{IN}_{3}(u), \operatorname{IN}_{4}(u)\right): \mathrm{U} \longrightarrow[0$, $1]$, and $\mathrm{FN}_{\tilde{Y}}(u)=\left(\mathrm{FN}_{1}(u), \mathrm{FN}_{2}(u), \mathrm{FN}_{3}(u), \mathrm{FN}_{4}(u)\right): \mathrm{U}$ $\longrightarrow \quad[0, \quad 1] \quad$ with the condition $0 \leq \mathrm{TN}_{4}(u)+\mathrm{IN}_{4}(u)+\mathrm{FN}_{4}(u) \leq 3$ for $u \in U$. For convenience, a $\operatorname{TrNN}$ in $\widetilde{Y}$ is simply denoted by $\widetilde{y}=<(\mathrm{TN} 1, \mathrm{TN} 2$, TN3, TN4), (IN1, IN2, IN3, IN4), (FN1, FN2, FN3, FN4)>.

Regarding two TrNNs $\tilde{y}_{1}=<\left(T N_{11}, T N_{12}, T N_{13}, T N_{14}\right)$, $\left(I N_{11}, I N_{12}, I N_{13}, I N_{14}\right),\left(F N_{11}, F N_{12}, F N_{13}, F N_{14}\right)>$ and $\tilde{y}_{2}=$ $<\left(T N_{21}, T N_{22}, T N_{23}, T N_{24}\right),\left(I N_{21}, I N_{22}, I N_{23}, I N_{24}\right),\left(F N_{21}\right.$, $\left.F N_{22}, F N_{23}, F N_{24}\right)>$, Ye [14] defined the following basic operations:

(1) $\widetilde{y}_{1} \oplus \widetilde{y}_{2}=\left\langle\left(T N_{11}+T N_{21}-T N_{11} T N_{21}, \quad T N_{12}+\right.\right.$ $T N_{22}-T N_{12} T N_{22}, \quad T N_{13}+T N_{23}-T N_{13} T N_{23}$, $\left.T N_{14}+T N_{24}-T N_{14} T N_{24}\right),\left(I N_{11} I N_{21}, I N_{12} I N_{22}\right.$, $\left.I N_{13} I N_{23}, I N_{14} I N_{24}\right), \quad\left(F N_{11} F N_{21}, F N_{12} F N_{22}\right.$, $\left.\left.F N_{13} F N_{23}, F N_{14} F N_{24}\right)\right\rangle$

(2) $\tilde{y}_{1} \otimes \tilde{y}_{2}=\left\langle\left(T N_{11} T N_{21}, T N_{12} T N_{22}, \quad T N_{13} T N_{23}\right.\right.$, $\left.T N_{14} T N_{24}\right),\left(I N_{11}+I N_{21}-I N_{11} I N_{21}, I N_{12}+\right.$ $I N_{22}-I N_{12} I N_{22}, I N_{13}+I N_{23}-I N_{13} I N_{23}, I N_{14}+$ $\left.I N_{24}-I N_{14} I N_{24}\right),\left(F N_{11}+F N_{21}-F N_{11} F N_{21}\right.$, $F N_{12}+F N_{22}-F N_{12} F N_{22}, F N_{13}+F N_{23}-F N_{13}$ $\left.\left.F N_{23}, F N_{14}+F N_{24}-F N_{14} F N_{24}\right)\right\rangle$

(3) $\lambda \widetilde{y}_{1}=\left\langle\left(1-\left(1-T N_{11}\right)^{\lambda}, 1-\left(1-T N_{12}\right)^{\lambda}, 1-(1-\right.\right.$ $\left.\left.T N_{13}\right)^{\lambda}, 1-\left(1-T N_{14}\right)^{\lambda}\right),\left(I N_{11}^{\lambda}, I N_{12}^{\lambda}, I N_{13}^{\lambda}, I N_{14}^{\lambda}\right)$, $\left.\left(F N_{11}^{\lambda}, F N_{12}^{\lambda}, F N_{13}^{\lambda}, F N_{14}^{\lambda}\right)\right\rangle, \lambda>0$

(4) $\tilde{y}_{1}^{\lambda}=\left\langle\left(T N_{11}^{\lambda}, T N_{12}^{\lambda}, T N_{13}^{\lambda}, T N_{14}^{\lambda}\right),\left(1-\left(1-I N_{11}\right)^{\lambda}\right.\right.$, $\left.1-\left(1-I N_{12}\right)^{\lambda}, 1-\left(1-I N_{13}\right)^{\lambda}, \quad 1-\left(1-I N_{14}\right)^{\lambda}\right)$, $\left(1-\left(1-F N_{11}\right)^{\lambda}, 1-\left(1-F N_{12}\right)^{\lambda}, 1-\left(1-F N_{13}\right)^{\lambda}\right.$, $\left.\left.1-\left(1-F N_{14}\right)^{\lambda}\right)\right\rangle, \lambda \geq 0$

Regarding a group of $\operatorname{TrNNs} \tilde{y}_{j}=<\left(T N_{j 1}, T N_{j 2}, T N_{j 3}\right.$, $\left.T N_{j 4}\right),\left(I N_{j 1}, I N_{j 2}, I N_{j 3}, I N_{j 4}\right),\left(F N_{j 1}, F N_{j 2}, F N_{j 3}, F N_{j 4}\right)>(j=1$, $2, \ldots, n)$ with their weights $\lambda_{j}(j=1,2, \ldots, n)$ for $\lambda_{j} \in[0,1]$ and $\sum_{j=1}^{n} \lambda_{j}=1$, Ye [9] proposed the TrNNWAA and TrNNWGA operators: 


$$
\begin{aligned}
\operatorname{Tr} & \operatorname{NWAA}\left(\tilde{y}_{1}, \tilde{y}_{2}, \ldots, \tilde{y}_{n}\right)=\bigoplus_{j=1}^{n} \lambda_{j} \tilde{y}_{j} \\
= & \left\langle\left(1-\prod_{j=1}^{n}\left(1-T N_{j 1}\right)^{\lambda_{j}}, 1-\prod_{j=1}^{n}\left(1-T N_{j 2}\right)^{\lambda_{j}}, 1-\prod_{j=1}^{n}\left(1-T N_{j 3}\right)^{\lambda_{j}}, 1-\prod_{j=1}^{n}\left(1-T N_{j 4}\right)^{\lambda_{j}}\right),\right. \\
& \left.\left(\prod_{j=1}^{n} I N_{j 1}^{\lambda_{j}}, \prod_{j=1}^{n} I N_{j 2}^{\lambda_{j}}, \prod_{j=1}^{n} I N_{j 3}^{\lambda_{j}}, \prod_{j=1}^{n} I N_{j 4}^{\lambda_{j}}\right),\left(\prod_{j=1}^{n} F N_{j 1}^{\lambda_{j}}, \prod_{j=1}^{n} F N_{j 2}^{\lambda_{j}}, \prod_{j=1}^{n} F N_{j 3}^{\lambda_{j}}, \prod_{j=1}^{n} F N_{j 4}^{\lambda_{j}}\right)\right\rangle, \\
\operatorname{TrNNWGA}\left(\tilde{y}_{1}, \tilde{y}_{2}, \ldots, \tilde{y}_{n}\right)=\prod_{j=1}^{n} \tilde{y}_{j}^{\lambda_{j}} & \left\langle\left(\prod_{j=1}^{n} T N_{j 1}^{\lambda_{j}}, \prod_{j=1}^{n} T N_{j 2}^{\lambda_{j}}, \prod_{j=1}^{n} T N_{j 3}^{\lambda_{j}}, \prod_{j=1}^{n} T N_{j 4}^{\lambda_{j}}\right),\right. \\
& \left(1-\prod_{j=1}^{n}\left(1-I N_{j 1}\right)^{\lambda_{j}}, 1-\prod_{j=1}^{n}\left(1-I N_{j 2}\right)^{\lambda_{j}}, 1-\prod_{j=1}^{n}\left(1-I N_{j 3}\right)^{\lambda_{j}}, 1-\prod_{j=1}^{n}\left(1-I N_{j 4}\right)^{\lambda_{j}}\right), \\
& \left.\left(1-\prod_{j=1}^{n}\left(1-F N_{j 1}\right)^{\lambda_{j}}, 1-\prod_{j=1}^{n}\left(1-F N_{j 2}\right)^{\lambda_{j}}, 1-\prod_{j=1}^{n}\left(1-F N_{j 3}\right)^{\lambda_{j}}, 1-\prod_{j=1}^{n}\left(1-F N_{j 4}\right)^{\lambda_{j}}\right)\right\rangle .
\end{aligned}
$$

Then, the score and accuracy functions of the $\operatorname{TrNN} \tilde{y}=$ $<\left(T N_{1}, T N_{2}, T N_{3}, T N_{4}\right),\left(I N_{1}, I N_{2}, I N_{3}, I N_{4}\right),\left(F N_{1}, F N_{2}, F N_{3}\right.$, $\mathrm{FN}_{4}$ ) > were defined as follows [9]:

$$
\begin{gathered}
S(\tilde{y})=\frac{1}{3}\left(2+\frac{T N_{1}+T N_{2}+T N_{3}+T N_{4}}{4}-\frac{I N_{1}+I N_{2}+I N_{3}+I N_{4}}{4}-\frac{F N_{1}+F N_{2}+F N_{3}+F N_{4}}{4}\right), \quad S(\tilde{y}) \in[0,1], \\
H(\tilde{y})=\frac{T N_{1}+T N_{2}+T N_{3}+T N_{4}}{4}-\frac{F N_{1}+F N_{2}+F N_{3}+F N_{4}}{4}, \quad H(\tilde{y}) \in[-1,1] .
\end{gathered}
$$

Based on the score and accuracy functions of $\operatorname{TrNNs}$, the ranking relations between two TrNNs $\tilde{y}_{1}=<\left(T N_{11}, T N_{12}\right.$, $\left.T N_{13}, T N_{14}\right),\left(I N_{11}, I N_{12}, I N_{13}, I N_{14}\right),\left(F N_{11}, F N_{12}, F N_{13}\right.$, $\left.F N_{14}\right)>$ and $\tilde{y}_{2}=<\left(T N_{21}, T N_{22}, T N_{23}, T N_{24}\right),\left(I N_{21}, I N_{22}\right.$, $\left.I N_{23}, I N_{24}\right),\left(F N_{21}, F N_{22}, F N_{23}, F N_{24}\right)>$ were defined as follows [9]:

(1) $\tilde{y}_{1}>\widetilde{y}_{2}$ for $S\left(\widetilde{y}_{1}\right)=S\left(\widetilde{y}_{2}\right)$

(2) $\tilde{y}_{1}>\widetilde{y}_{2}$ for $S\left(\widetilde{y}_{1}\right)=S\left(\widetilde{y}_{2}\right)$ and $H\left(\widetilde{y}_{1}\right)>H\left(\tilde{y}_{2}\right)$

(3) $\tilde{y}_{1} \cong \tilde{y}_{2}$ for $S\left(\widetilde{y}_{1}\right)=S\left(\widetilde{y}_{2}\right)$ and $H\left(\widetilde{y}_{1}\right)=H\left(\widetilde{y}_{2}\right)$

\section{Trapezoidal Neutrosophic Z-Number (TrNZN) Sets}

To make trapezoidal neutrosophic information reliable, this section gives the following definitions of a TrNZN set, operations of TrNZNs, score and accuracy functions of TrNZN, and ranking laws of TrNZNs.

Definition 1. Set $U$ as a universe set; then, a TrNZN set in $U$ is defined as the following mathematical representation:

$$
\widetilde{Z}=\left\{u,\left\langle\left(T Z_{\widetilde{V}}(u), T Z_{\widetilde{R}}(u)\right),\left(I Z_{\widetilde{V}}(u), I Z_{\widetilde{R}}(u)\right),\left(F Z_{\widetilde{V}}(u), F Z_{\widetilde{R}}(u)\right)\right\rangle \mid u \in U\right\},
$$

where $\left(T Z_{\widetilde{V}}(u), T Z_{\widetilde{R}}(u)\right),\left(I Z_{\widetilde{V}}(u), I Z_{\widetilde{R}}(u)\right)$, and $\left(F Z_{\widetilde{V}}(u)\right.$, $\left.F Z_{\widetilde{R}}(u)\right)$ are the truth, indeterminacy, and falsity trapezoidal Z-numbers that are composed of the truth, indeterminacy, and falsity trapezoidal fuzzy numbers and their reliability measures, denoted as $\left(T Z_{\widetilde{V}}(u), T Z_{\widetilde{R}}(u)\right)=\left(\left(T_{V 1}(u), T_{V 2}(u), T_{V 3}(u)\right.\right.$, $\left.\left.T_{V 4}(u)\right),\left(T_{R 1}(u), T_{R 2}(u), T_{R 3}(u), T_{R 4}(u)\right)\right): U \longrightarrow[0,1] \times$ $[0,1],\left(\left(I Z_{\widetilde{V}}(u), I Z_{\widetilde{R}}(u)=\left(\left(I_{V 1}(u), I_{V 2}(u), I_{V 3}(u), I_{V 4}(u)\right)\right.\right.\right.$,
$\left.\left(I_{R 1}(u), I_{R 2}(u), I_{R 3}(u), I_{R 4}(u)\right)\right): U \longrightarrow[0,1] \times[0,1]$, and $\left(F Z_{\widetilde{V}}(u), F Z_{\widetilde{R}}(u)\right)=\left(\left(F_{V 1}(u), F_{V 2}(u), F_{V 3}(u), F_{V 4}(u)\right),\left(F_{R 1}\right.\right.$ $\left.\left.(u), F_{R 2}(u), \stackrel{R}{F}_{R 3}(u), F_{R 4}(u)\right)\right): U \longrightarrow[0,1] \times[0,1]$ with the conditions $0 \leq T_{V 4}(u)+I_{V 4}(u)+F_{V 4}(u) \leq 3$ and $0 \leq T_{R 4}(u)+$ $I_{R 4}(u)+F_{R 4}(u) \leq 3$ for $u \in U$.

For convenience, the three trapezoidal Z-numbers in $\widetilde{Z}$ are simply denoted as $\left(T Z_{\widetilde{V}}(u), T Z_{\widetilde{R}}(u)\right)=\left(\left(T_{V 1}\right.\right.$, 
$\left.\left.T_{V 2}, T_{V 3}, T_{V 4}\right),\left(T_{R 1}, T_{R 2}, T_{R 3}, T_{R 4}\right)\right), \quad\left(I Z_{\widetilde{V}}(u), I Z_{\widetilde{R}}(u)\right)=$ $\left(\left(I_{V 1}, I_{V 2}, I_{V 3}, I_{V 4}\right),\left(I_{R 1}, I_{R 2}, I_{R 3}, I_{R 4}\right)\right)$, and $\left(F Z_{\widetilde{V}}(u), F Z_{\widetilde{R}}\right.$ $(u))=\left(\left(F_{V 1}, F_{V 2}, F_{V 3}, F_{V 4}\right),\left(F_{R 1}, F_{R 2}, F_{R 3}, F_{R 4}\right)\right)$. Thus, a $\operatorname{TrNZN}$ in $\widetilde{Z}$ is simply denoted as $\widetilde{z}=<\left(\left(T_{V 1}, T_{V 2}, T_{V 3}, T_{V 4}\right)\right.$, $\left.\left(T_{R 1}, T_{R 2}, T_{R 3}, T_{R 4}\right)\right),\left(\left(I_{V 1}, I_{V 2}, I_{V 3}, I_{V 4}\right),\left(I_{R 1}, I_{R 2}, I_{R 3}, T_{R 4}\right)\right)$, $\left(\left(F_{V 1}, F_{V 2}, F_{V 3}, F_{V 4}\right),\left(F_{R 1}, F_{R 2}, F_{R 3}, F_{R 4}\right)\right)>$.

If $T_{V 2}=T_{V 3}, T_{R 2}=T_{R 3}, I_{V 2}=I_{V 3}, I_{R 2}=I_{R 3}$, and $F_{V 2}=F_{V 3}$, $F_{R 2}=F_{R 3}$ hold in the TrNZN $\widetilde{z}$; it is reduced to the triangular neutrosophic Z-number, which is a special case of TrNZN.

Definition 2. Set $\widetilde{z}_{1}=<\left(\left(T_{V 11}, T_{V 12}, T_{V 13}, T_{V 14}\right),\left(T_{R 11}, T_{R 12}\right.\right.$, $\left.\left.T_{R 13}, T_{R 14}\right)\right),\left(\left(I_{V 11}, I_{V 12}, I_{V 13}, I_{V 14}\right),\left(I_{R 11}, I_{R 12}, I_{R 13}, T_{R 14}\right)\right)$, $\left(\left(F_{V 11}, F_{V 12}, F_{V 13}, F_{V 14}\right),\left(F_{R 11}, F_{R 12}, F_{R 13}, F_{R 14}\right)\right)>$ and $\widetilde{z}_{2}=$ $<\left(\left(T_{V 21}, T_{V 22}, T_{V 23}, T_{V 24}\right),\left(T_{R 21}, T_{R 22}, T_{R 23}, T_{R 24}\right)\right),\left(\left(I_{V 21}\right.\right.$, $\left.\left.I_{V 22}, I_{V 23}, I_{V 24}\right),\left(I_{R 21}, I_{R 22}, I_{R 23}, T_{R 24}\right)\right),\left(\left(F_{V 21}, F_{V 22}, F_{V 23}\right.\right.$, $\left.\left.F_{V 24}\right),\left(F_{R 21}, F_{R 22}, F_{R 23}, F_{R 24}\right)\right)>$ as two TrNZNs. Then they are defined as the following basic operations:

(1) $\widetilde{z}_{1} \oplus \widetilde{z}_{2}=\left\langle\left(\left(T_{V 11}+T_{V 21}-T_{V 11} T_{V 21}, T_{V 12}+T_{V 22}-\right.\right.\right.$ $T_{V 12} T_{V 22}, T_{V 13}+T_{V 23}-T_{V 13} T_{V 23}, \quad T_{V 14}+T_{V 24}-$ $\left.T_{V 14} T_{V 24}\right),\left(T_{R 11}+T_{R 21}-T_{R 11} T_{R 21}, T_{R 12}+T_{R 22}-\right.$ $T_{R 12} T_{R 22}, \quad T_{R 13}+T_{R 23}-T_{R 13} T_{R 23}, T_{R 14}+T_{R 24}-$ $\left.\left.T_{R 14} T_{R 24}\right)\right),\left(\left(I_{V 11} I_{V 21}, \quad I_{V 12} I_{V 22}, I_{V 13} I_{V 23}, I_{V 14} I_{V 24}\right)\right.$, $\left.\left(I_{R 11} I_{R 21}, I_{R 12} I_{R 22}, I_{R 13} I_{R 23}, I_{R 14} I_{R 24}\right)\right), \quad\left(\left(F_{V 11} F_{V 21}\right.\right.$, $\left.F_{V 12} F_{V 22}, F_{V 13} F_{V 23}, F_{V 14} F_{V 24}\right),\left(F_{R 11} F_{R 21}, F_{R 12} F_{R 22}\right.$, $\left.\left.\left.F_{R 13} F_{R 23}, F_{R 14} F_{R 24}\right)\right)\right\rangle$

(2) $\tilde{z}_{1} \otimes \widetilde{z}_{2}=\left\langle\left(\left(T_{V 11} T_{V 21}, \quad T_{V 12} T_{V 22}, T_{V 13} T_{V 23}, T_{V 14}\right.\right.\right.$ $\left.\left.T_{V 24}\right)\left(T_{R 11} T_{R 21}, T_{R 12} T_{R 22}, \quad T_{R 13} T_{R 23}, T_{R 14} T_{R 24}\right)\right)$, $\left(\left(I_{V 11}+I_{V 21}-I_{V 11} I_{V 21}, \quad I_{V 12}+I_{V 22}-I_{V 12} I_{V 22}\right.\right.$, $\left.I_{V 13}+I_{V 23}-I_{V 13} I_{V 23}, I_{V 14}+I_{V 24}-I_{V 14} I_{V 24}\right),\left(I_{R 11}+\right.$ $I_{R 21}-I_{R 11} I_{R 21}, \quad I_{R 12}+I_{R 22}-I_{R 12} I_{R 22}, I_{R 13}+I_{R 23}-$
$\left.\left.I_{R 13} I_{R 23}, I_{R 14}+I_{R 24}-I_{R 14} I_{R 24}\right)\right), \quad\left(\left(F_{V 11}+F_{V 21}-\right.\right.$ $F_{V 11} F_{V 21}, F_{V 12}+F_{V 22}-F_{V 12} F_{V 22}, F_{V 13}+F_{V 23}-F_{V 13}$ $\left.F_{V 23}, F_{V 14}+F_{V 24}-F_{V 14} F_{V 24}\right), \quad\left(F_{R 11}+F_{R 21}-F_{R 11}\right.$ $F_{R 21}, F_{R 12}+F_{R 22}-F_{R 12} F_{R 22}, F_{R 13}+F_{R 23}-F_{R 13} F_{R 23}$, $\left.\left.\left.F_{R 14}+F_{R 24}-F_{R 14} F_{R 24}\right)\right)\right\rangle$

(3) $\lambda \widetilde{z}_{1}=\left\langle\left(\left(1-\left(1-T_{V 11}\right)^{\lambda}, \quad 1-\left(1-T_{V 12}\right)^{\lambda}, 1-(1-\right.\right.\right.$ $\left.\left.T_{V 13}\right)^{\lambda}, 1-\left(1-T_{V 14}\right)^{\lambda}\right),\left(1-\left(1-T_{R 11}\right)^{\lambda}, \quad 1-(1-\right.$ $\left.\left.\left.T_{R 12}\right)^{\lambda}, 1-\left(1-T_{R 13}\right)^{\lambda}, 1-\left(1-T_{R 14}\right)^{\lambda}\right)\right),\left(\left(I_{V 11}^{\lambda}, I_{V 12}^{\lambda}\right.\right.$, $\left.\left.I_{V 13}^{\lambda}, I_{V 14}^{\lambda}\right),\left(I_{R 11}^{\lambda}, I_{R 12}^{\lambda}, I_{R 13}^{\lambda}, \quad I_{R 14}^{\lambda}\right)\right),\left(\left(F_{V 11}^{\lambda}, F_{V 12}^{\lambda}\right.\right.$, $\left.\left.\left.F_{V 13}^{\lambda}, F_{V 14}^{\lambda}\right),\left(F_{R 11}^{\lambda}, F_{R 12}^{\lambda}, F_{R 13}^{\lambda}, F_{R 14}^{\lambda}\right)\right)\right\rangle, \lambda>0$

(4) $\tilde{z}_{1}^{\lambda}=\left\langle\left(\left(T_{V 11}^{\lambda}, T_{V 12}^{\lambda}, T_{V 13}^{\lambda}, T_{V 14}^{\lambda}\right), \quad\left(T_{R 11}^{\lambda}, T_{R 12}^{\lambda}, T_{R 13}^{\lambda}\right.\right.\right.$, $\left.\left.T_{R 14}^{\lambda}\right)\right),\left(\left(1-\left(1-I_{V 11}\right)^{\lambda}, \quad 1-\left(1-I_{V 12}\right)^{\lambda}, 1-(1-\right.\right.$ $\left.\left.I_{V 13}\right)^{\lambda}, \quad 1-\left(1-I_{V 14}\right)^{\lambda}\right),\left(1-\left(1-I_{R 11}\right)^{\lambda}, 1-(1-\right.$ $\left.\left.\left.I_{R 12}\right)^{\lambda}, 1-\left(1-I_{R 13}\right)^{\lambda}, \quad 1-\left(1-I_{R 14}\right)^{\lambda}\right)\right),((1-(1-$ $\left.F_{V 11}\right)^{\lambda}, 1-\left(1-F_{V 12}\right)^{\lambda}, 1-\left(1-F_{V 13}\right)^{\lambda}, \quad 1-(1-$ $\left.\left.F_{V 14}\right)^{\lambda}\right),\left(1-\left(1-F_{R 11}\right)^{\lambda}, \quad 1-\left(1-F_{R 12}\right)^{\lambda}, 1-(1-\right.$ $\left.\left.\left.\left.F_{R 13}\right)^{\lambda}, 1-\left(1-F_{R 14}\right)^{\lambda}\right)\right)\right\rangle, \lambda>0$

For ranking TrNZNs, the score and accuracy functions of TrNZN are defined according to the expected value of a trapezoidal fuzzy number and score and accuracy functions of $\operatorname{TrNN}[9]$.

Definition 3. Set $\widetilde{z}_{1}=<\left(\left(T_{V 11}, T_{V 12}, T_{V 13}, T_{V 14}\right),\left(T_{R 11}, T_{R 12}\right.\right.$, $\left.\left.T_{R 13}, T_{R 14}\right)\right),\left(\left(I_{V 11}, I_{V 12}, I_{V 13}, I_{V 14}\right),\left(I_{R 11}, I_{R 12}, I_{R 13}, T_{R 14}\right)\right)$, $\left(\left(F_{V 11}, F_{V 12}, F_{V 13}, F_{V 14}\right),\left(F_{R 11}, F_{R 12}, F_{R 13}, F_{R 14}\right)\right)>$ as TrNZN. Then the score and accuracy functions of the $\operatorname{TrNZN} \widetilde{z}_{1}$ can be defined as follows:

$$
\begin{aligned}
& S\left(\widetilde{z}_{1}\right)=\frac{1}{3}\left(\begin{array}{c}
2+\frac{T_{V 11}+T_{V 12}+T_{V 13}+T_{V 14}}{4} \times \frac{T_{R 11}+T_{R 12}+T_{R 13}+T_{R 14}}{4} \\
-\frac{I_{V 11}+I_{V 12}+I_{V 13}+I_{V 14}}{4} \times \frac{I_{R 11}+I_{R 12}+I_{R 13}+I_{R 14}}{4} \\
-\frac{F_{V 11}+F_{V 12}+F_{V 13}+F_{V 14}}{4} \times \frac{F_{R 11}+F_{R 12}+F_{R 13}+F_{R 14}}{4}
\end{array}\right), \quad S\left(\widetilde{z}_{1}\right) \in[0,1], \\
& H\left(\widetilde{z}_{1}\right)= \frac{T_{V 11}+T_{V 12}+T_{V 13}+T_{V 14}}{4} \times \frac{T_{R 11}+T_{R 12}+T_{R 13}+T_{R 14}}{4}-\frac{F_{V 11}+F_{V 12}+F_{V 13}+F_{V 14}}{4} \\
& \times \frac{F_{R 11}+F_{R 12}+F_{R 13}+F_{R 14}}{4}, \quad H(\widetilde{z}) \in[-1,1] .
\end{aligned}
$$

Based on equations (7) and (8), ranking laws between two TrNZNs are given by the following definition.

Definition 4. Set $\tilde{z}_{1}=<\left(\left(T_{V 11}, T_{V 12}, T_{V 13}, T_{V 14}\right),\left(T_{R 11}, T_{R 12}\right.\right.$, $\left.\left.T_{R 13}, T_{R 14}\right)\right),\left(\left(I_{V 11}, I_{V 12}, I_{V 13}, I_{V 14}\right),\left(I_{R 11}, I_{R 12}, I_{R 13}, T_{R 14}\right)\right)$, $\left(\left(F_{V 11}, F_{V 12}, F_{V 13}, F_{V 14}\right),\left(F_{R 11}, F_{R 12}, F_{R 13}, F_{R 14}\right)\right)>$ and $\widetilde{z}_{2}=$ $<\left(\left(T_{V 21}, T_{V 22}, T_{V 23}, T_{V 24}\right),\left(T_{R 21}, T_{R 22}, T_{R 23}, T_{R 24}\right)\right),\left(\left(I_{V 21}\right.\right.$, $\left.\left.I_{V 22}, I_{V 23}, I_{V 24}\right),\left(I_{R 21}, I_{R 22}, I_{R 23}, T_{R 24}\right)\right),\left(\left(F_{V 21}, F_{V 22}, F_{V 23}\right.\right.$, $\left.\left.F_{V 24}\right),\left(F_{R 21}, F_{R 22}, F_{R 23}, F_{R 24}\right)\right)>$ as two TrNZNs. Then, the ranking laws between two TrNZNs are defined as follows:
(1) If $\mathrm{S}\left(\widetilde{z}_{1}\right)>\mathrm{S}\left(\widetilde{z}_{2}\right)$, then $\widetilde{z}_{1}>\widetilde{z}_{2}$

(2) If $S\left(\widetilde{z}_{1}\right)=S\left(\widetilde{z}_{2}\right)$ and $H\left(\widetilde{z}_{1}\right)>H\left(\widetilde{z}_{2}\right)$, then $\widetilde{z}_{1}>\widetilde{z}_{2}$

(3) If $S\left(\widetilde{z}_{1}\right)=S\left(\widetilde{z}_{2}\right)$ and $H\left(\widetilde{z}_{1}\right)=H\left(\widetilde{z}_{2}\right)$, then $\widetilde{z}_{1} \cong \widetilde{z}_{2}$

\section{Weighted Aggregation Operators of TrNZNs}

Regarding information aggregation in MDM problems, one usually utilizes the weighted arithmetic and geometric averaging operators as the most basic information aggregation 
approaches. To aggregate TrNZNs, therefore, this section proposes the two following weighted aggregation operators of TrNZNs based on the basic operations of TrNZNs in Definition 2.

\subsection{Weighted Arithmetic Averaging Operator of TrNZNs}

Definition 5. Set $\widetilde{z}_{j}=<\left(\left(T_{V j 1}, T_{V j 2}, T_{V j 3}, T_{V j 4}\right),\left(T_{R j 1}, T_{R j 2}\right.\right.$, $\left.\left.T_{R j 3}, T_{R j 4}\right)\right),\left(\left(I_{V j 1}, I_{V j 2}, I_{V j 3}, I_{V j 4}\right),\left(I_{R j 1}, I_{R j 2}, I_{R j 3}, I_{R j 4}\right)\right),\left(\left(F_{V j 1}\right.\right.$, $\left.\left.F_{V j 2}, F_{V j 3}, F_{V j 4}\right),\left(F_{R j 1}, F_{R j 2}, F_{R j 3}, F_{R j 4}\right)\right)>(j=1,2, \ldots, n)$ as a series of TrNZNs. Then, the TrNZNWAA operator is defined as

$$
\operatorname{TrNZNWAA}\left(\widetilde{z}_{1}, \widetilde{z}_{2}, \ldots, \widetilde{z}_{n}\right)=\bigoplus_{j=1}^{n} \lambda_{j} \widetilde{z}_{j}
$$

where $\lambda_{j}(j=1,2, \ldots, n)$ is the weight of the $j$ th $\operatorname{TrNZN} \widetilde{z}_{j}$ $(j=1,2, \ldots, n)$ for $\lambda_{j} \in[0,1]$ and $\sum_{j=1}^{n} \lambda_{j}=1$.

Based on the basic operations of TrNZNs in Definition 2 and equation (9), we have the following theorem.

Theorem 1. Set $\tilde{z}_{j}=<\left(\left(T_{V j 1}, T_{V j 2}, T_{V j 3}, T_{V j 4}\right),\left(T_{R j 1}, T_{R j 2}\right.\right.$, $\left.\left.T_{R j 3}, T_{R j 4}\right)\right),\left(\left(I_{V j 1}, I_{V j 2}, I_{V j 3}, I_{V j 4}\right),\left(I_{R j 1}, I_{R j 2}, I_{R j 3}, I_{R j 4}\right)\right),\left(\left(F_{V j 1}\right.\right.$, $\left.\left.F_{V j 2}, F_{V j 3}, F_{V j 4}\right),\left(F_{R j 1}, F_{R j 2}, F_{R j 3}, F_{R j 4}\right)\right)>(j=1,2, \ldots, n)$ as a series of TrNZNs. Then, the aggregated value of equation (9) is also TrNZN, which is yielded by the following equation:

$$
\begin{array}{r}
\operatorname{TrNZNWAA}\left(\widetilde{z}_{1}, \tilde{z}_{2}, \ldots, \tilde{z}_{n}\right) \\
=\left\langle\begin{array}{r}
\left(\left(1-\prod_{j=1}^{n}\left(1-T_{V j 1}\right)^{\lambda_{j}}, 1-\prod_{j=1}^{n}\left(1-T_{V j 2}\right)^{\lambda_{j}}, 1-\prod_{j=1}^{n}\left(1-T_{V j 3}\right)^{\lambda_{j}}, 1-\prod_{j=1}^{n}\left(1-T_{V j 4}\right)^{\lambda_{j}}\right),\left(1-\prod_{j=1}^{n}\left(1-T_{R j 1}\right)^{\lambda_{j}}, 1-\prod_{j=1}^{n}\left(1-T_{R j 2}\right)^{\lambda_{j}}, 1-\prod_{j=1}^{n}\left(1-T_{R j 3}\right)^{\lambda_{j}}, 1-\prod_{j=1}^{n}\left(1-T_{R j 4}\right)^{\lambda_{j}}\right)\right), \\
\quad\left(\left(\prod_{j=1}^{n} I_{V j 1}^{\lambda_{j}}, \prod_{j=1}^{n} I_{V j 2}^{\lambda_{j}}, \prod_{j=1}^{n} I_{V j 3}^{\lambda_{j}}, \prod_{j=1}^{n} I_{V j 4}^{\lambda_{j}}\right),\left(\prod_{j=1}^{n} I_{R j 1}^{\lambda_{j}}, \prod_{j=1}^{n} I_{R j 2}^{\lambda_{j}}, \prod_{j=1}^{n} I_{R j 3}^{\lambda_{j}}, \prod_{j=1}^{n} I_{R j 4}^{\lambda_{j}}\right)\right),\left(\left(\prod_{j=1}^{n} F_{V j 1}^{\lambda_{j}}, \prod_{j=1}^{n} F_{V j 2}^{\lambda_{j}}, \prod_{j=1}^{n} F_{V j 3}^{\lambda_{j}}, \prod_{j=1}^{n} F_{V j 4}^{\lambda_{j}}\right),\left(\prod_{j=1}^{n} F_{R j 1}^{\lambda_{j}}, \prod_{j=1}^{n} F_{R j 2}^{\lambda_{j}}, \prod_{j=1}^{n} F_{R j 3}^{\lambda_{j}}, \prod_{j=1}^{n} F_{R j 4}^{\lambda_{j}}\right)\right)
\end{array}\right.
\end{array}
$$

where $\lambda_{j}(j=1,2, \ldots, n)$ is the weight of the $j$ th $\operatorname{TrNZN} \widetilde{z}_{j}(j=1$, $2, \ldots, n)$ for $\lambda_{j} \in[0,1]$ and $\sum_{j=1}^{n} \lambda_{j}=1$.
Proof. The proof of equation (10) can be given by mathematical induction.

(1) Set $n=2$. Then there is the following result:

$$
\begin{aligned}
& \operatorname{TrNZNWAA}\left(\widetilde{z}_{1}, \widetilde{z}_{2}\right)=\lambda_{1} \widetilde{z}_{1} \oplus \lambda_{2} \widetilde{z}_{2} \\
& =\left\langle\left(\left( 1-\left(1-T_{V 11}\right)^{\lambda_{1}}+1-\left(1-T_{V 21}\right)^{\lambda_{2}}-\left(1-\left(1-T_{V 11}\right)^{\lambda_{1}}\right)\left(1-\left(1-T_{V 21}\right)^{\lambda_{2}}\right),\right.\right.\right. \\
& 1-\left(1-T_{V 12}\right)^{\lambda_{1}}+1-\left(1-T_{V 22}\right)^{\lambda_{2}}-\left(1-\left(1-T_{V 12}\right)^{\lambda_{1}}\right)\left(1-\left(1-T_{V 22}\right)^{\lambda_{2}}\right) \text {, } \\
& 1-\left(1-T_{V 13}\right)^{\lambda_{1}}+1-\left(1-T_{V 23}\right)^{\lambda_{2}}-\left(1-\left(1-T_{V 13}\right)^{\lambda_{1}}\right)\left(1-\left(1-T_{V 23}\right)^{\lambda_{2}}\right) \text {, } \\
& \left.1-\left(1-T_{V 14}\right)^{\lambda_{1}}+1-\left(1-T_{V 24}\right)^{\lambda_{2}}-\left(1-\left(1-T_{V 14}\right)^{\lambda_{1}}\right)\left(1-\left(1-T_{V 24}\right)^{\lambda_{2}}\right)\right) \text {, } \\
& \left(1-\left(1-T_{R 11}\right)^{\lambda_{1}}+1-\left(1-T_{R 21}\right)^{\lambda_{2}}-\left(1-\left(1-T_{R 11}\right)^{\lambda_{1}}\right)\left(1-\left(1-T_{R 21}\right)^{\lambda_{2}}\right)\right. \text {, } \\
& 1-\left(1-T_{R 12}\right)^{\lambda_{1}}+1-\left(1-T_{R 22}\right)^{\lambda_{2}}-\left(1-\left(1-T_{R 12}\right)^{\lambda_{1}}\right)\left(1-\left(1-T_{R 22}\right)^{\lambda_{2}}\right) \text {, } \\
& 1-\left(1-T_{R 13}\right)^{\lambda_{1}}+1-\left(1-T_{R 23}\right)^{\lambda_{2}}-\left(1-\left(1-T_{R 13}\right)^{\lambda_{1}}\right)\left(1-\left(1-T_{R 23}\right)^{\lambda_{2}}\right) \text {, } \\
& \left.\left.1-\left(1-T_{V 14}\right)^{\lambda_{1}}+1-\left(1-T_{R 24}\right)^{\lambda_{2}}-\left(1-\left(1-T_{R 14}\right)^{\lambda_{1}}\right)\left(1-\left(1-T_{R 24}\right)^{\lambda_{2}}\right)\right)\right) \text {, } \\
& \left(\left(I_{V 11}^{\lambda_{1}} I_{V 21}^{\lambda_{2}}, I_{V 12}^{\lambda_{1}} I_{V 22}^{\lambda_{2}}, I_{V 13}^{\lambda_{1}} I_{V 23}^{\lambda_{2}}, I_{V 14}^{\lambda_{1}} I_{V 24}^{\lambda_{2}}\right),\left(I_{R 11}^{\lambda_{1}} I_{R 21}^{\lambda_{2}}, I_{R 12}^{\lambda_{1}} I_{R 22}^{\lambda_{2}}, I_{R 13}^{\lambda_{1}} I_{R 23}^{\lambda_{2}}, I_{R 14}^{\lambda_{1}} I_{R 24}^{\lambda_{2}}\right)\right) \text {, } \\
& \left.\left(\left(F_{V 11}^{\lambda_{1}} F_{V 21}^{\lambda_{2}}, F_{V 12}^{\lambda_{1}} F_{V 22}^{\lambda_{2}}, F_{V 13}^{\lambda_{1}} F_{V 23}^{\lambda_{2}}, F_{V 14}^{\lambda_{1}} F_{V 24}^{\lambda_{2}}\right),\left(F_{R 11}^{\lambda_{1}} F_{R 21}^{\lambda_{2}}, F_{R 12}^{\lambda_{1}} F_{R 22}^{\lambda_{2}}, F_{R 13}^{\lambda_{1}} F_{R 23}^{\lambda_{2}}, F_{R 14}^{\lambda_{1}} F_{R 24}^{\lambda_{2}}\right)\right)\right\rangle \\
& =\left\langle\left(\left( 1-\left(1-T_{V 11}\right)^{\lambda_{1}}\left(1-T_{V 21}\right)^{\lambda_{2}}, 1-\left(1-T_{V 12}\right)^{\lambda_{1}}\left(1-T_{V 22}\right)^{\lambda_{2}}\right.\right.\right. \text {, } \\
& \left.1-\left(1-T_{V 13}\right)^{\lambda_{1}}\left(1-T_{V 23}\right)^{\lambda_{2}}, 1-\left(1-T_{V 14}\right)^{\lambda_{1}}\left(1-T_{V 24}\right)^{\lambda_{2}}\right) \\
& \left(1-\left(1-T_{R 11}\right)^{\lambda_{1}}\left(1-T_{R 21}\right)^{\lambda_{2}}, 1-\left(1-T_{R 12}\right)^{\lambda_{1}}\left(1-T_{R 22}\right)^{\lambda_{2}}\right. \text {, } \\
& \left.\left.1-\left(1-T_{R 13}\right)^{\lambda_{1}}\left(1-T_{R 23}\right)^{\lambda_{2}}, 1-\left(1-T_{R 14}\right)^{\lambda_{1}}\left(1-T_{R 24}\right)^{\lambda_{2}}\right)\right) \text {, } \\
& \left(\left(\prod_{j=1}^{2} I_{V j 1}^{\lambda_{j}}, \prod_{j=1}^{2} I_{V j 2}^{\lambda_{j}}, \prod_{j=1}^{2} I_{V j 3}^{\lambda_{j}}, \prod_{j=1}^{2} I_{V j 4}^{\lambda_{j}}\right),\left(\prod_{j=1}^{2} I_{R j 1}^{\lambda_{j}}, \prod_{j=1}^{2} I_{R j 2}^{\lambda_{j}}, \prod_{j=1}^{2} I_{R j 3}^{\lambda_{j}}, \prod_{j=1}^{2} I_{R j 4}^{\lambda_{j}}\right)\right), \\
& \left.\left(\left(\prod_{j=1}^{2} F_{V j 1}^{\lambda_{j}}, \prod_{j=1}^{2} F_{V j 2}^{\lambda_{j}}, \prod_{j=1}^{2} F_{V j 3}^{\lambda_{j}}, \prod_{j=1}^{2} F_{V j 4}^{\lambda_{j}}\right),\left(\prod_{j=1}^{2} F_{R j 1}^{\lambda_{j}}, \prod_{j=1}^{2} F_{R j 2}^{\lambda_{j}}, \prod_{j=1}^{2} F_{R j 3}^{\lambda_{j}}, \prod_{j=1}^{2} F_{R j 4}^{\lambda_{j}}\right)\right)\right\rangle .
\end{aligned}
$$


(2) Set $n=k$. Then, equation (10) can hold in the following equation:

$$
\begin{aligned}
& \operatorname{Tr} \operatorname{NZNWAA}\left(\tilde{z}_{1}, \tilde{z}_{2}, \ldots, \widetilde{z}_{k}\right)=\bigoplus_{j=1}^{k} \lambda_{j} \widetilde{z}_{j} \\
&=\left\langle\left(\left(1-\prod_{j=1}^{k}\left(1-T_{V j 1}\right)^{\lambda_{j}}, 1-\prod_{j=1}^{k}\left(1-T_{V j 2}\right)^{\lambda_{j}}, 1-\prod_{j=1}^{k}\left(1-T_{V j 3}\right)^{\lambda_{j}}, 1-\prod_{j=1}^{k}\left(1-T_{V j 4}\right)^{\lambda_{j}}\right),\right.\right. \\
& \\
&\left.\left(1-\prod_{j=1}^{k}\left(1-T_{R j 1}\right)^{\lambda_{j}}, 1-\prod_{j=1}^{k}\left(1-T_{R j 2}\right)^{\lambda_{j}}, 1-\prod_{j=1}^{k}\left(1-T_{R j 3}\right)^{\lambda_{j}}, 1-\prod_{j=1}^{k}\left(1-T_{R j 4}\right)^{\lambda_{j}}\right)\right), \\
&\left(\left(\prod_{j=1}^{k} I_{V j 1}^{\lambda_{j}}, \prod_{j=1}^{k} I_{V j 2}^{\lambda_{j}}, \prod_{j=1}^{k} I_{V j 3}^{\lambda_{j}}, \prod_{j=1}^{k} I_{V j 4}^{\lambda_{j}}\right),\left(\prod_{j=1}^{k} I_{R j 1}^{\lambda_{j}}, \prod_{j=1}^{k} I_{R j 2}^{\lambda_{j}}, \prod_{j=1}^{k} I_{R j 3}^{\lambda_{j}}, \prod_{j=1}^{k} I_{R j 4}^{\lambda_{j}}\right)\right), \\
&\left.\left(\left(\prod_{j=1}^{k} F_{V j 1}^{\lambda_{j}}, \prod_{j=1}^{k} F_{V j 2}^{\lambda_{j}}, \prod_{j=1}^{k} F_{V j 3}^{\lambda_{j}}, \prod_{j=1}^{k} F_{V j 4}^{\lambda_{j}}\right),\left(\prod_{j=1}^{k} F_{R j 1}^{\lambda_{j}}, \prod_{j=1}^{k} F_{R j 2}^{\lambda_{j}}, \prod_{j=1}^{k} F_{R j 3}^{\lambda_{j}}, \prod_{j=1}^{k} F_{R j 4}^{\lambda_{j}}\right)\right)\right\rangle .
\end{aligned}
$$

(3) Set $n=k+1$. By equations (11) and (12), we can obtain

$\operatorname{TrNZNWAA}\left(\tilde{z}_{1}, \tilde{z}_{2}, \ldots, \tilde{z}_{k}, \tilde{z}_{k+1}\right)=\underset{j=1}{\stackrel{\oplus}{\oplus}} \lambda_{j} \tilde{z}_{j} \oplus \lambda_{k+1} \tilde{z}_{k+1}$

$$
\begin{aligned}
& \left.\begin{array}{l}
\left(\begin{array}{l}
\left.1-\prod_{j=1}^{k}\left(1-T_{V j 1}\right)^{\lambda_{j}}+1-\left(1-T_{V(k+1) 1}\right)\right)^{\lambda_{k+1}}-\left(\left(1-\prod_{j=1}^{k}\left(1-T_{V j 1}\right)^{\lambda_{j}}\right)\left(1-\left(1-T_{V(k+1) 1}\right)\right)^{\lambda_{k+1}}\right), 1-\prod_{j=1}^{k}\left(1-T_{V j 2}\right)^{\lambda_{j}}+1-\left(1-T_{V(k+1) 2}\right)^{\lambda_{k+1}}-\left(\left(1-\prod_{j=1}^{k}\left(1-T_{V j 2}\right)^{\lambda_{j}}\right)\left(1-\left(1-T_{V(k+1) 2}\right)\right)^{\lambda_{k+1}}\right), \\
1-\prod_{j=1}^{k}\left(1-T_{V j 3}\right)^{\lambda_{j}}+1-\left(1-T_{V(k+1) 3}\right)^{\lambda_{k+1}}-\left(\left(1-\prod_{j=1}^{k}\left(1-T_{V j 3}\right)^{\lambda_{j}}\right)\left(1-\left(1-T_{V(k+1) 3}\right)\right)^{\lambda_{k+1}}\right), 1-\prod_{j=1}^{k}\left(1-T_{V j 4}\right)^{\lambda_{j}}+1-\left(1-T_{V(k+1) 4}\right)^{\lambda_{k+1}}-\left(\left(1-\prod_{j=1}^{k}\left(1-T_{V j 4}\right)^{\lambda_{j}}\right)\left(1-\left(1-T_{V(k+1) 4}\right)\right)^{\lambda_{k+1}}\right)
\end{array}\right) \\
=\left\langle\left(\begin{array}{l}
\left(1-\prod_{j=1}^{k}\left(1-T_{R j 1}\right)^{\lambda_{j}}+1-\left(1-T_{R(k+1) 1}\right)^{\lambda_{k+1}}-\left(\left(1-\prod_{j=1}^{k}\left(1-T_{R j 1}\right)^{\lambda_{j}}\right)\left(1-\left(1-T_{R(k+1) 1}\right)\right)^{\lambda_{k+1}}\right), 1-\prod_{j=1}^{k}\left(1-T_{R j 2}\right)^{\lambda_{j}}+1-\left(1-T_{R(k+1) 2}\right)^{\lambda_{k+1}}-\left(\left(1-\prod_{j=1}^{k}\left(1-T_{R j 2}\right)^{\lambda_{j}}\right)\left(1-\left(1-T_{R(k+1) 2}\right)\right)^{\lambda_{k+1}}\right),\right. \\
1-\prod_{j=1}^{k}\left(1-T_{R j 3}\right)^{\lambda_{j}}+1-\left(1-T_{R(k+1) 3}\right)^{\lambda_{k+1}}-\left(\left(1-\prod_{j=1}^{k}\left(1-T_{R j 3}\right)^{\lambda_{j}}\right)\left(1-\left(1-T_{R(k+1) 3}\right)^{\lambda_{k+1}}\right), 1-\prod_{j=1}^{k}\left(1-T_{R j 4}\right)^{\lambda_{j}}+1-\left(1-T_{R(k+1) 4}\right)^{\lambda_{k+1}}-\left(\left(1-\prod_{j=1}^{k}\left(1-T_{R j 4}\right)^{\lambda_{j}}\right)\left(1-\left(1-T_{R(k+1) 4}\right)^{\lambda_{k+1}}\right),\right.\right.
\end{array}\right)\right\rangle
\end{array}\right)
\end{aligned}
$$

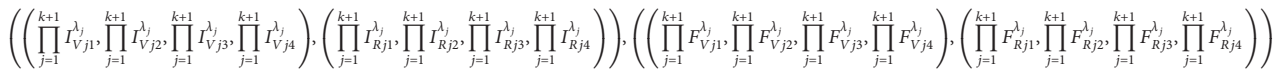

$$
\begin{aligned}
& =\left\langle\left(\left(1-\prod_{j=1}^{k+1}\left(1-T_{V j 1}\right)^{\lambda_{j}}, 1-\prod_{j=1}^{k+1}\left(1-T_{V j 2}\right)^{\lambda_{j}}, 1-\prod_{j=1}^{k+1}\left(1-T_{V j 3}\right)^{\lambda_{j}}, 1-\prod_{j=1}^{k+1}\left(1-T_{V j 4}\right)^{\lambda_{j}},\right)\left(1-\prod_{j=1}^{k+1}\left(1-T_{R j 1}\right)^{\lambda_{j}}, 1-\prod_{j=1}^{k+1}\left(1-T_{R j 2}\right)^{\lambda_{j},}, 1-\prod_{j=1}^{k+1}\left(1-T_{R j 3}\right)^{\lambda_{j}}, 1-\prod_{j=1}^{k+1}\left(1-T_{R j 4}\right)^{\lambda_{j}}\right)\right),{ }^{k+1}\right\rangle
\end{aligned}
$$

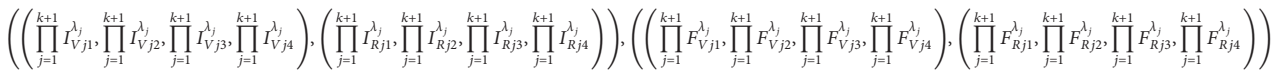

Regarding the above results, equation (10) can hold for any $n$. Thus, the proof is completed.

Especially when $\lambda_{j}=1 / n(j=1,2, \ldots, n)$, the TrNZNWAA operator is reduced to the TrNZN arithmetic averaging operator.

Theorem 2. The TrNZNWAA operator contains the three following properties:
(P1) Idempotency: set $\widetilde{z}_{j}=<\left(\left(T_{V j 1}, T_{V j 2}, T_{V j 3}, T_{V j 4}\right)\right.$, $\left.\left(T_{R j 1}, T_{R j 2}, T_{R j 3}, T_{R j 4}\right)\right),\left(\left(I_{V j 1}, I_{V j 2}, I_{V j 3}, I_{V j 4}\right),\left(I_{R j 1}, I_{R j 2}\right.\right.$, $\left.\left.I_{R j 3}, I_{R j 4}\right)\right),\left(\left(F_{V j 1}, F_{V j 2}, F_{V j 3}, F_{V j 4}\right),\left(F_{R j 1}, F_{R j 2}, F_{R j 3}, F_{R j 4}\right)\right)$ $>(j=1,2, \ldots, n)$ as a series of $\operatorname{TrNZNs}$. If $\widetilde{z}_{j}=\widetilde{z}$ for $j=1$, $2, \ldots, n$, then there exists $\operatorname{TrNZNWAA}\left(\widetilde{z}_{1}, \widetilde{z}_{2}, \ldots\right.$, $\left.\widetilde{z}_{n}\right)=\widetilde{z}$

(P2) Set $\widetilde{z}_{j}=<\left(\left(T_{V j 1}, T_{V j 2}, T_{V j 3}, T_{V j 4}\right),\left(T_{R j 1}, T_{R j 2}, T_{R j 3}\right.\right.$, $\left.\left.T_{R j 4}\right)\right),\left(\left(I_{V j 1}, I_{V j 2}, I_{V j 3}, I_{V j 4}\right),\left(I_{R j 1}, I_{R j 2}, I_{R j 3}, I_{R j 4}\right)\right),\left(\left(F_{V j 1}\right.\right.$, 
$\left.\left.F_{V j 2}, F_{V j 3}, F_{V j 4}\right),\left(F_{R j 1}, F_{R j 2}, F_{R j 3}, F_{R j 4}\right)\right)>(j=1,2, \ldots, n)$ as a series of TrNZNs; then, set the minimum and maximum TrNZNs as

$$
\begin{aligned}
&\left(\left(\min _{j} T_{V j 1}, \min _{j} T_{V j 2}, \min _{j} T_{V j 3}, \min _{j} T_{V j 4}\right),\left(\min _{j} T_{R j 1}, \min _{j} T_{R j 2}, \min _{j} T_{R j 3}, \min _{j} T_{R j 4}\right)\right), \\
& \tilde{z}^{-}=\left\langle\left(\left(\max _{j} I_{V j 1}, \max _{j} I_{V j 2}, \max _{j} I_{V j 3}, \max _{j} I_{V j 4}\right),\left(\max _{j} I_{R j 1}, \max _{j} I_{R j 2}, \max _{j} I_{R j 3}, \max _{j} I_{R j 4}\right)\right),\right\rangle, \\
&\left(\left(\max _{j} F_{V j 1}, \max _{j} F_{V j 2}, \max _{j} F_{V j 3}, \max _{j} F_{V j 4}\right),\left(\max _{j} F_{R j 1}, \max _{j} F_{R j 2}, \max _{j} F_{R j 3}, \max _{j} F_{R j 4}\right)\right) \\
&\left(\left(\max _{j} T_{V j 1}, \max _{j} T_{V j 2}, \max _{j} T_{V j 3}, \max _{j} T_{V j 4}\right),\left(\max _{j} T_{R j 1}, \max _{j} T_{R j 2}, \max _{j} T_{R j 3}, \max _{j} T_{R j 4}\right)\right), \\
& \tilde{z}^{+}=\left\langle\left(\left(\min _{j} I_{V j 1}, \min _{j} I_{V j 2}, \min _{j} I_{V j 3}, \min _{j} I_{V j 4}\right),\left(\min _{j} I_{R j 1}, \min _{j} I_{R j 2}, \min _{j} I_{R j 3}, \min _{j} I_{R j 4}\right)\right),\right\rangle, \\
&\left.\left(\left(\min _{j} F_{V j 1}, \min _{j} F_{V j 2}, \min _{j} F_{V j 3}, \min _{j} F_{V j 4}\right),\left(\min _{j} F_{R j 1}, \min _{j} F_{R j 2}, \min _{j} F_{R j 3}, \min _{j} F_{R j 4}\right)\right)\right\rangle
\end{aligned}
$$

Then, there is $\widetilde{z}^{-} \leq \operatorname{TrNZNWAA}\left(\widetilde{z}_{1}, \widetilde{z}_{2}, \ldots, \widetilde{z}_{n}\right) \leq \widetilde{z}^{+}$. Proof.

(P3) Monotony: set $\widetilde{z}_{j}=<((T V j 1, T V j 2, T V j 3, T V j 4)$, (TRj1, TRj2, TRj3, TRj4)), ((IVj1, IVj2, IVj3, IVj4), (IRj1, IRj2, IRj3, IRj4)), ((FVj1, FVj2, FVj3, FVj4), $(F R j 1, F R j 2, F R j 3, F R j 4))>(j=1,2, \ldots, n)$ as a series of TrNZNs. If $\widetilde{z}_{j} \leq \widetilde{z}_{j}^{*}$ for $j=1,2, \ldots, n$, then there is $\operatorname{TrNZNWAA}\left(\widetilde{z}_{1}, \widetilde{z}_{2}, \ldots, \widetilde{z}_{n}\right) \leq \operatorname{TrNZNWAA} \quad\left(\widetilde{z}_{1}^{*}\right.$, $\left.\widetilde{z}_{2}^{*}, \ldots, \widetilde{z}_{n}^{*}\right)$.
(P1) Owing to $\widetilde{z}_{j}=\widetilde{z}$ for $j=1,2, \ldots, n$, there is the following result:

$$
\begin{aligned}
& \operatorname{TrNZNWAA}\left(\widetilde{z}_{1}, \widetilde{z}_{2}, \ldots, \widetilde{z}_{n}\right)=\underset{j=1}{\oplus} \lambda_{j} \widetilde{z}_{j} \\
& =\left\langle\left(\left(1-\prod_{j=1}^{n}\left(1-T_{V j 1}\right)^{\lambda_{j}}, 1-\prod_{j=1}^{n}\left(1-T_{V j 2}\right)^{\lambda_{j}}, 1-\prod_{j=1}^{n}\left(1-T_{V j 3}\right)^{\lambda_{j}}, 1-\prod_{j=1}^{n}\left(1-T_{V j 4}\right)^{\lambda_{j}}\right),\right.\right. \\
& \left(1-\prod_{j=1}^{n}\left(1-T_{R j 1}\right)^{\lambda_{j}}, 1-\prod_{j=1}^{n}\left(1-T_{R j 2}\right)^{\lambda_{j}}, 1-\prod_{j=1}^{n}\left(1-T_{R j 3}\right)^{\lambda_{j}}, 1-\prod_{j=1}^{n}\left(1-T_{R j 4}\right)^{\lambda_{j}}\right), \\
& \left(\left(\prod_{j=1}^{n} I_{V j 1}^{\lambda_{j}}, \prod_{j=1}^{n} I_{V j 2}^{\lambda_{j}}, \prod_{j=1}^{n} I_{V j 3}^{\lambda_{j}}, \prod_{j=1}^{n} I_{V j 4}^{\lambda_{j}}\right),\left(\prod_{j=1}^{n} I_{R j 1}^{\lambda_{j}}, \prod_{j=1}^{n} I_{R j 2}^{\lambda_{j}}, \prod_{j=1}^{n} I_{R j 3}^{\lambda_{j}}, \prod_{j=1}^{n} I_{R j 4}^{\lambda_{j}}\right)\right), \\
& \left.\left(\left(\prod_{j=1}^{n} F_{V j 1}^{\lambda_{j}}, \prod_{j=1}^{n} F_{V j 2}^{\lambda_{j}}, \prod_{j=1}^{n} F_{V j 3}^{\lambda_{j}}, \prod_{j=1}^{n} F_{V j 4}^{\lambda_{j}}\right),\left(\prod_{j=1}^{n} F_{R j 1}^{\lambda_{j}}, \prod_{j=1}^{n} F_{R j 2}^{\lambda_{j}}, \prod_{j=1}^{n} F_{R j 3}^{\lambda_{j}}, \prod_{j=1}^{n} F_{R j 4}^{\lambda_{j}}\right)\right)\right\rangle \\
& =\left\langle\left(\left(1-\left(1-T_{V 1}\right)_{j=1} n \lambda_{j}, 1-\left(1-T_{V 2}\right)_{j=1} n \lambda_{j}, 1-\left(1-T_{V 3}\right)_{j=1} n \lambda_{j}, 1-\left(1-T_{V 4}\right)_{j=1} n \lambda_{j}\right),\right.\right. \\
& \left.\left(1-\left(1-T_{R 1}\right) \sum_{j=1} n \lambda_{j}, 1-\left(1-T_{R 2}\right)_{j=1} n \lambda_{j}, 1-\left(1-T_{R 3}\right)_{j=1} n \lambda_{j}, 1-\left(1-T_{R 4}\right)_{j=1} n \lambda_{j}\right)\right),
\end{aligned}
$$




$$
\begin{aligned}
& \left(\left(I_{V 1} j=1^{n} w_{j}, I_{V 2} j=1^{n} w_{j}, I_{V 3} j=1^{n} w_{j}, I_{V 4} j=1^{n} w_{j}\right),\right. \\
& \left.\left(I_{R 1} j=1^{n} w_{j}, I_{R 2} j=1^{n} w_{j}, I_{R 3} j=1^{n} w_{j}, I_{R 4} j=1^{n} w_{j}\right)\right), \\
& \left(\left(F_{V 1} j=1^{n} w_{j}, F_{V 2} j=1^{n} w_{j}, F_{V 3} j=1^{n} w_{j}, F_{V 4} j=1^{n} w_{j}\right),\right. \\
& \left.\left.\left(F_{R 1} j=1^{n} w_{j}, F_{R 2} j=1^{n} w_{j}, F_{R 3} j=1^{n} w_{j}, F_{R 4} j=1^{n} w_{j}\right)\right)\right\rangle \\
= & \left\langle\left(\left(T_{V 1}, T_{V 2}, T_{V 3}, T_{V 4}\right),\left(T_{R 1}, T_{R 2}, T_{R 3}, T_{R 4}\right)\right),\left(\left(I_{V 1}, I_{V 2}, I_{V 3}, I_{V 4}\right),\left(I_{R 1}, I_{R 2}, I_{R 3}, I_{R 4}\right)\right),\right. \\
& \left.\left(\left(I_{V 1}, I_{V 2}, I_{V 3}, I_{V 4}\right),\left(I_{R 1}, I_{R 2}, I_{R 3}, I_{R 4}\right)\right)\right\rangle=\widetilde{z} .
\end{aligned}
$$

(P2) Due to $\widetilde{z}^{-} \leq \widetilde{z}_{j} \leq \widetilde{z}^{+}$for $j=1,2, \ldots, n$, there exists $\oplus_{j=1}^{n} \lambda_{j} \widetilde{z}^{-} \leq \oplus_{j=1}^{n} \lambda_{j} \tilde{z}_{j} \leq \oplus_{j=1}^{n} \lambda_{j} \tilde{z}^{+}$. So, the inequality $\widetilde{z}^{-} \leq \Theta_{j=1}^{n} \lambda_{j} \widetilde{z}_{j} \leq \widetilde{z}^{+}$can hold according to (P1); that is, $\widetilde{z}^{-} \leq \operatorname{TrNZNWAA}\left(\widetilde{z}_{1}, \widetilde{\tilde{n}}_{2}, \ldots, \widetilde{\tilde{n}}_{n}\right) \leq \widetilde{z}^{+}$.

(P3) Due to $\widetilde{z}_{j} \leq \widetilde{z}_{j}^{*}$ for $j=1,2, \ldots, n$, there is $\oplus_{j=1}^{n} \lambda_{j} \widetilde{z}_{j}$ $\leq \oplus_{j=1}^{n} \lambda_{j} \widetilde{z}_{j}^{*}$; that is, $\operatorname{TrNZNWAA}\left(\widetilde{z}_{1}, \widetilde{z}_{2}, \ldots, \widetilde{z}_{n}\right) \leq$ $\operatorname{TrNZNWAA}\left(\widetilde{z}_{1}^{*}, \widetilde{z}_{2}^{*}, \ldots, \widetilde{z}_{n}^{*}\right)$.

Thus, the proof of these properties is completed.

\subsection{Weighted Geometric Averaging Operator of TrNZNs}

Definition 6. Set $\widetilde{z}_{j}=<\left(\left(T_{V j 1}, T_{V j 2}, T_{V j 3}, T_{V j 4}\right),\left(T_{R j 1}, T_{R j 2}\right.\right.$, $\left.\left.T_{R j 3}, T_{R j 4}\right)\right),\left(\left(I_{V j 1}, I_{V j 2}, I_{V j 3}, I_{V j 4}\right),\left(I_{R j 1}, I_{R j 2}, I_{R j 3}, I_{R j 4}\right)\right),\left(\left(F_{V j 1}\right.\right.$, $\left.\left.F_{V j 2}, F_{V j 3}, F_{V j 4}\right),\left(F_{R j 1}, F_{R j 2}, F_{R j 3}, F_{R j 4}\right)\right)>(j=1,2, \ldots, n)$ as a series of TrNZNs. Then, the TrNZNWGA operator is defined as

$$
\operatorname{TrNZNWGA}\left(\widetilde{z}_{1}, \widetilde{z}_{2}, \ldots, \widetilde{z}_{n}\right)=\bigotimes_{j=1}^{n} \widetilde{z}_{j}^{\lambda_{j}},
$$

where $\lambda_{j}(j=1,2, \ldots, n)$ is the weight of the $j$ th $\operatorname{TrNZN} \widetilde{z}_{j}$ for $\lambda_{j} \in[0,1]$ and $\sum_{j=1}^{n} \lambda_{j}=1$.

Regarding the basic operations of TrNZNs in Definition 2 and equation (16), we can give the theorem below.

Theorem 3. Set $\widetilde{z}_{j}=<\left(\left(T_{V j 1}, T_{V j 2}, T_{V j 3}, T_{V j 4}\right),\left(T_{R j 1}, T_{R j 2}\right.\right.$, $\left.\left.T_{R j 3}, T_{R j 4}\right)\right),\left(\left(I_{V j 1}, I_{V j 2}, I_{V j 3}, I_{V j 4}\right),\left(I_{R j 1}, I_{R j 2}, I_{R j 3}, I_{R j 4}\right)\right),\left(\left(F_{V j 1}\right.\right.$, $\left.\left.F_{V j 2}, F_{V j 3}, F_{V j 4}\right),\left(F_{R j 1}, F_{R j 2}, F_{R j 3}, F_{R j 4}\right)\right)>(j=1,2, \ldots, n)$ as a series of TrNZNs. Then, the aggregated value of the TrNZNWGA operator is also TrNZN, which is obtained by

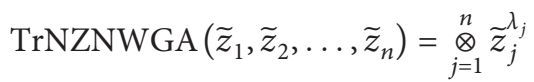

$$
\begin{aligned}
& =\left\langle\left(\left(\prod_{j=1}^{n} T_{V j 1}^{\lambda_{j}}, \prod_{j=1}^{n} T_{V j 2}^{\lambda_{j}}, \prod_{j=1}^{n} T_{V j 3}^{\lambda_{j}}, \prod_{j=1}^{n} T_{V j 4}^{\lambda_{j}}\right),\left(\prod_{j=1}^{n} T_{R j 1}^{\lambda_{j}}, \prod_{j=1}^{n} T_{R j 2}^{\lambda_{j}}, \prod_{j=1}^{n} T_{R j 3}^{\lambda_{j}}, \prod_{j=1}^{n} T_{R j 4}^{\lambda_{j}}\right)\right),\right. \\
& \left(\left(1-\prod_{j=1}^{n}\left(1-I_{V j 1}\right)^{\lambda_{j}}, 1-\prod_{j=1}^{n}\left(1-I_{V j 2}\right)^{\lambda_{j}}, 1-\prod_{j=1}^{n}\left(1-I_{V j 3}\right)^{\lambda_{j}}, 1-\prod_{j=1}^{n}\left(1-I_{V j 4}\right)^{\lambda_{j}}\right),\right. \\
& \left.\left(1-\prod_{j=1}^{n}\left(1-I_{R j 1}\right)^{\lambda_{j}}, 1-\prod_{j=1}^{n}\left(1-I_{R j 2}\right)^{\lambda_{j}}, 1-\prod_{j=1}^{n}\left(1-I_{R j 3}\right)^{\lambda_{j}}, 1-\prod_{j=1}^{n}\left(1-I_{R j 4}\right)^{\lambda_{j}}\right)\right), \\
& \left(\left(1-\prod_{j=1}^{n}\left(1-F_{V j 1}\right)^{\lambda_{j}}, 1-\prod_{j=1}^{n}\left(1-F_{V j 2}\right)^{\lambda_{j}}, 1-\prod_{j=1}^{n}\left(1-F_{V j 3}\right)^{\lambda_{j}}, 1-\prod_{j=1}^{n}\left(1-F_{V j 4}\right)^{\lambda_{j}}\right),\right. \\
& \left.\left.\left(1-\prod_{j=1}^{n}\left(1-F_{R j 1}\right)^{\lambda_{j}}, 1-\prod_{j=1}^{n}\left(1-F_{R j 2}\right)^{\lambda_{j}}, 1-\prod_{j=1}^{n}\left(1-F_{R j 3}\right)^{\lambda_{j}}, 1-\prod_{j=1}^{n}\left(1-F_{R j 4}\right)^{\lambda_{j}}\right)\right)\right\rangle .
\end{aligned}
$$


where $\lambda_{j}(j=1,2, \ldots, n)$ is the weight of the $j$ th $\operatorname{TrNZN} \widetilde{z}_{j}$ for $\lambda_{j} \in[0,1]$ and $\sum_{j=1}^{n} \lambda_{j}=1$.

Based on the similar proof process of Theorem 1, we can verify Theorem 3, which is omitted.

In particular, the TrNZNWGA operator is reduced to the $\operatorname{TrNZN}$ geometric averaging operator when $\lambda_{j}=1 / n(j=1,2$, ..., n).

Theorem 4. The TrNZNWGA operator also contains the three following properties:
(P1) Idempotency: set $\widetilde{z}_{j}=<\left(\left(T_{V j 1}, T_{V j 2}, T_{V j 3}, T_{V j 4}\right)\right.$, $\left.\left(T_{R j 1}, T_{R j 2}, T_{R j 3}, T_{R j 4}\right)\right),\left(\left(I_{V j 1}, I_{V j 2}, I_{V j 3}, I_{V j 4}\right),\left(I_{R j 1}, I_{R j 2}\right.\right.$, $\left.\left.I_{R j 3}, I_{R j 4}\right)\right),\left(\left(F_{V j 1}, F_{V j 2}, F_{V j 3}, F_{V j 4}\right),\left(F_{R j 1}, F_{R j 2}, F_{R j 3}, F_{R j 4}\right)\right)$ $>(j=1,2, \ldots, n)$ as a series of $\operatorname{Tr} N Z N$ s. If $\widetilde{z}_{j}=\widetilde{z}$ for $j=1$, $2, \ldots, n$, then there exists $\operatorname{TrNZNWGA}\left(\widetilde{z}_{1}, \widetilde{z}_{2}, \ldots, \widetilde{z}_{n}\right)=$ $\tilde{z}$.

(P2) Boundedness: set $\widetilde{z}_{j}=<\left(\left(T_{V j 1}, T_{V j 2}, T_{V j 3}, T_{V j 4}\right)\right.$, $\left.\left(T_{R j 1}, T_{R j 2}, T_{R j 3}, T_{R j 4}\right)\right),\left(\left(I_{V j 1}, I_{V j 2}, I_{V j 3}, I_{V j 4}\right),\left(I_{R j 1}, I_{R j 2}\right.\right.$, $\left.\left.I_{R j 3}, I_{R j 4}\right)\right),\left(\left(F_{V j 1}, F_{V j 2}, F_{V j 3}, F_{V j 4}\right),\left(F_{R j 1}, F_{R j 2}, F_{R j 3}, F_{R j 4}\right)\right)$ $>(j=1,2, \ldots, n)$ as a series of TrNZNs; then set the minimum and maximum TrNZNs as

$$
\begin{aligned}
& \left(\left(\min _{j} T_{V j 1}, \min _{j} T_{V j 2}, \min _{j} T_{V j 3}, \min _{j} T_{V j 4}\right),\left(\min _{j} T_{R j 1}, \min _{j} T_{R j 2}, \min _{j} T_{R j 3}, \min _{j} T_{R j 4}\right)\right), \\
& \tilde{z}^{-}=\left\langle\left(\left(\max _{j} I_{V j 1}, \max _{j} I_{V j 2}, \max _{j} I_{V j 3}, \max _{j} I_{V j 4}\right),\left(\max _{j} I_{R j 1}, \max _{j} I_{R j 2}, \max _{j} I_{R j 3}, \max _{j} I_{R j 4}\right)\right), \quad\right\rangle, \\
& \left(\left(\max _{j} F_{V j 1}, \max _{j} F_{V j 2}, \max _{j} F_{V j 3}, \max _{j} F_{V j 4}\right),\left(\max _{j} F_{R j 1}, \max _{j} F_{R j 2}, \max _{j} F_{R j 3}, \max _{j} F_{R j 4}\right)\right) \\
& \left(\left(\max _{j} T_{V j 1}, \max _{j} T_{V j 2}, \max _{j} T_{V j 3}, \max _{j} T_{V j 4}\right),\left(\max _{j} T_{R j 1}, \max _{j} T_{R j 2}, \max _{j} T_{R j 3}, \max _{j} T_{R j 4}\right)\right), \\
& \widetilde{z}^{+}=\left\langle\left(\left(\min _{j} I_{V j 1}, \min _{j} I_{V j 2}, \min _{j} I_{V j 3}, \min _{j} I_{V j 4}\right),\left(\min _{j} I_{R j 1}, \min _{j} I_{R j 2}, \min _{j} I_{R j 3}, \min _{j} I_{R j 4}\right)\right),\right\rangle \\
& \left(\left(\min _{j} F_{V j 1}, \min _{j} F_{V j 2}, \min _{j} F_{V j 3}, \min _{j} F_{V j 4}\right),\left(\min _{j} F_{R j 1}, \min _{j} F_{R j 2}, \min _{j} F_{R j 3}, \min _{j} F_{R j 4}\right)\right)
\end{aligned}
$$

Then, there is $\widetilde{z}^{-} \leq \operatorname{TrNZNWGA}\left(\widetilde{z}_{1}, \widetilde{z}_{2}, \ldots, \widetilde{z}_{n}\right) \leq \widetilde{z}^{+}$. (P3) Monotony: set $\widetilde{z}_{j}=<\left(\left(T_{V j 1}, T_{V j 2}, T_{V j 3}, T_{V j 4}\right),\left(T_{R j 1}\right.\right.$, $\left.\left.T_{R j 2}, T_{R j 3}, T_{R j 4}\right)\right),\left(\left(I_{V j 1}, I_{V j 2}, I_{V j 3}, I_{V j 4}\right),\left(I_{R j 1}, I_{R j 2}, I_{R j 3}\right.\right.$, $\left.\left.I_{R j 4}\right)\right),\left(\left(F_{V j 1}, F_{V j 2}, F_{V j 3}, F_{V j 4}\right),\left(F_{R j 1}, F_{R j 2}, F_{R j 3}, F_{R j 4}\right)\right)>$ $(j=1,2, \ldots, n)$ as a series of $\operatorname{TrNZNs}$. If $\widetilde{z}_{j} \leq \widetilde{z}_{j}^{*}$ for $j=1$, $2, \ldots, n$, then there exists TrNZNWGA $\left(\widetilde{z}_{1}, \widetilde{z}_{2}, \ldots, \widetilde{z}_{n}\right) \leq$ $\operatorname{TrNZNWGA}\left(\widetilde{z}_{1}^{*}, \widetilde{z}_{2}^{*}, \ldots, \widetilde{z}_{n}^{*}\right)$.

By the same proof process of Theorem 2, the properties of the TrNZNWGA operator can be also verified, which are not repeated here.

\section{MDM Approach Using the TrNZNWAA and TrNZNWGA Operators and Score and Accuracy Functions}

This section establishes an MDM approach by using the TrNZNWAA and TrNZNWGA operators and score and accuracy functions to handle MDM problems with TrNZN information.

Regarding an MDM problem with TrNZN information, a set of alternatives $Q=\left\{Q_{1}, Q_{2}, \ldots, Q_{m}\right\}$ are commonly presented and satisfactorily assessed by a set of criteria $S=$ $\left\{s_{1}, s_{2}, \ldots, s_{n}\right\}$. Each alternative over criteria is assessed by decision makers and then their given assessment values are expressed in the form of TrNZNs $\widetilde{z}_{i j}=<\left(\left(T_{V i j 1}, T_{V i j 2}, T_{V i j 3}\right.\right.$, $\left.\left.T_{V i j 4}\right),\left(T_{R i j 1}, T_{R i j 2}, T_{R i j 3}, T_{R i j 4}\right)\right),\left(\left(I_{V i j 1}, I_{V i j 2}, I_{V i j 3}, I_{V i j 4}\right),\left(I_{R i j 1}\right.\right.$, $\left.\left.I_{R i j 2}, I_{R i j 3}, I_{R i j 4}\right)\right),\left(\left(F_{V i j 1}, F_{V i j 2}, F_{V i j 3}, F_{V i j 4}\right),\left(F_{R i j 1}, F_{R i j 2}, F_{R i j 3}\right.\right.$, $\left.\left.F_{R i j 4}\right)\right)>(j=1,2, \ldots, n ; i=1,2, \ldots, m)$, where $\left(T_{V i j 1}, T_{V i j 2}, T_{V i j 3}\right.$, $\left.T_{V i j 4}\right) \subseteq[0,1]$ and $\left(T_{R i j 1}, T_{R i j 2}, T_{R i j 3}, T_{R i j 4}\right) \subseteq[0,1]$ indicate the truth degrees and reliability measures of the alternative $Q_{i}$ over the criteria $s_{j},\left(I_{V i j 1}, I_{V i j 2}, I_{V i j 3}, I_{V i j 4}\right) \subseteq[0,1]$ and $\left(I_{R i j 1}, I_{R i j 2}, I_{R i j 3}\right.$, $\left.I_{R i j 4}\right) \subseteq[0,1]$ indicate the indeterminate degrees and reliability measures of the alternative $Q_{i}$ over the criteria $s_{j}$, and $\left(F_{V i j 1}\right.$, $\left.F_{V i j 2}, F_{V i j 3}, F_{V i j 4}\right) \subseteq[0,1]$ and $\left(F_{R i j 1}, F_{R i j 2}, F_{R i j 3}, F_{R i j 4}\right) \subseteq[0,1]$ indicate the falsity degrees and reliability measures of the alternative $Q_{i}$ over the criteria $s_{j}$, along with $0 \leq T_{V i j 4}+I_{V i j 4}+F_{V i j 4} \leq 3$ and $0 \leq T_{R i j 4}+I_{R i j 4}+F_{R i j 4} \leq 3$ for $j=1$, $2, \ldots, n$ and $i=1,2, \ldots, m$. Then, all the specified TrNZNs are constructed as their decision matrix $\widetilde{Z}=\left(\widetilde{z}_{i j}\right)_{m \times n}$.

Thus, the TrNZNWAA and TrNZNWGA operators and the score and accuracy functions can be applied to MDM problems with TrNZN information, and then their MDM approach can be indicated by the following procedures:

Step 1: the aggregated $\operatorname{TrNZN} \widetilde{z}_{i}$ for $Q_{i}(i=1,2, \ldots, m)$ is obtained by applying the TrNZNWAA or TrNZNWGA operator: 


$$
\begin{aligned}
& \widetilde{z}_{i}=\operatorname{TrNZNWAA}\left(\widetilde{z}_{i 1}, \widetilde{z}_{i 2}, \ldots, \widetilde{z}_{i n}\right)=\bigoplus_{j=1}^{n} \lambda_{j} \widetilde{z}_{i j} \\
& =\left\langle\left(\left(1-\prod_{j=1}^{n}\left(1-T_{V i j 1}\right)^{\lambda_{j}}, 1-\prod_{j=1}^{n}\left(1-T_{V i j 2}\right)^{\lambda_{j}}, 1-\prod_{j=1}^{n}\left(1-T_{V i j 3}\right)^{\lambda_{j}}, 1-\prod_{j=1}^{n}\left(1-T_{V i j 4}\right)^{\lambda_{j}}\right),\right.\right. \\
& \left.\left(1-\prod_{j=1}^{n}\left(1-T_{R i j 1}\right)^{\lambda_{j}}, 1-\prod_{j=1}^{n}\left(1-T_{R i j 2}\right)^{\lambda_{j}}, 1-\prod_{j=1}^{n}\left(1-T_{R i j 3}\right)^{\lambda_{j}}, 1-\prod_{j=1}^{n}\left(1-T_{R i j 4}\right)^{\lambda_{j}}\right)\right), \\
& \left(\left(\prod_{j=1}^{n} I_{V i j 1}^{\lambda_{j}}, \prod_{j=1}^{n} I_{V i j 2}^{\lambda_{j}}, \prod_{j=1}^{n} I_{V i j 3}^{\lambda_{j}}, \prod_{j=1}^{n} I_{V i j 4}^{\lambda_{j}}\right),\left(\prod_{j=1}^{n} I_{R i j 1}^{\lambda_{j}}, \prod_{j=1}^{n} I_{R i j 2}^{\lambda_{j}}, \prod_{j=1}^{n} I_{R i j 3}^{\lambda_{j}}, \prod_{j=1}^{n} I_{R i j 4}^{\lambda_{j}}\right)\right), \\
& \left.\left(\left(\prod_{j=1}^{n} F_{V i j 1}^{\lambda_{j}}, \prod_{j=1}^{n} F_{V i j 2}^{\lambda_{j}}, \prod_{j=1}^{n} F_{V i j 3}^{\lambda_{j}}, \prod_{j=1}^{n} F_{V i j 4}^{\lambda_{j}}\right),\left(\prod_{j=1}^{n} F_{R i j 1}^{\lambda_{j}}, \prod_{j=1}^{n} F_{R i j 2}^{\lambda_{j}}, \prod_{j=1}^{n} F_{R i j 3}^{\lambda_{j}}, \prod_{j=1}^{n} F_{R i j 4}^{\lambda_{j}}\right)\right)\right\rangle, \\
& \widetilde{z}_{i}=\operatorname{TrNZNWGA}\left(\widetilde{z}_{i 1}, \widetilde{z}_{i 2}, \ldots, \widetilde{z}_{\text {in }}\right)=\otimes_{j=1}^{n} \widetilde{z}_{i j}^{\lambda_{j}} \\
& =\left\langle\left(\left(\prod_{j=1}^{n} T_{V i j 1}^{\lambda_{j}}, \prod_{j=1}^{n} T_{V i j 2}^{\lambda_{j}}, \prod_{j=1}^{n} T_{V i j 3}^{\lambda_{j}}, \prod_{j=1}^{n} T_{V i j 4}^{\lambda_{j}}\right),\left(\prod_{j=1}^{n} T_{R i j 1}^{\lambda_{j}}, \prod_{j=1}^{n} T_{R i j 2}^{\lambda_{j}}, \prod_{j=1}^{n} T_{R i j 3}^{\lambda_{j}}, \prod_{j=1}^{n} T_{R i j 4}^{\lambda_{j}}\right)\right),\right. \\
& \left(\left(1-\prod_{j=1}^{n}\left(1-I_{V i j 1}\right)^{\lambda_{j}}, 1-\prod_{j=1}^{n}\left(1-I_{V i j 2}\right)^{\lambda_{j}}, 1-\prod_{j=1}^{n}\left(1-I_{V i j 3}\right)^{\lambda_{j}}, 1-\prod_{j=1}^{n}\left(1-I_{V i j 4}\right)^{\lambda_{j}}\right),\right. \\
& \left.\left(1-\prod_{j=1}^{n}\left(1-I_{R i j 1}\right)^{\lambda_{j}}, 1-\prod_{j=1}^{n}\left(1-I_{R i j 2}\right)^{\lambda_{j}}, 1-\prod_{j=1}^{n}\left(1-I_{R i j 3}\right)^{\lambda_{j}}, 1-\prod_{j=1}^{n}\left(1-I_{R i j 4}\right)^{\lambda_{j}}\right)\right), \\
& \left(\left(1-\prod_{j=1}^{n}\left(1-F_{V i j 1}\right)^{\lambda_{j}}, 1-\prod_{j=1}^{n}\left(1-F_{V i j 2}\right)^{\lambda_{j}}, 1-\prod_{j=1}^{n}\left(1-F_{V i j 3}\right)^{\lambda_{j}}, 1-\prod_{j=1}^{n}\left(1-F_{V i j 4}\right)^{\lambda_{j}}\right),\right. \\
& \left.\left.\left(1-\prod_{j=1}^{n}\left(1-F_{R i j 1}\right)^{\lambda_{j}}, 1-\prod_{j=1}^{n}\left(1-F_{R i j 2}\right)^{\lambda_{j}}, 1-\prod_{j=1}^{n}\left(1-F_{R i j 3}\right)^{\lambda_{j}}, 1-\prod_{j=1}^{n}\left(1-F_{R i j 4}\right)^{\lambda_{j}}\right)\right)\right\rangle .
\end{aligned}
$$

Step 2: by equation (7), we calculate the score values of $S\left(\widetilde{z}_{i}\right)$. If necessary, we calculate the accuracy values of $H\left(\widetilde{z}_{i}\right)(i=1,2, \ldots, m)$ by equation $(8)$.

Step 3: all the alternatives $Q_{i}(i=1,2, \ldots, m)$ are ranked corresponding to the score values (the accuracy values) and the best one(s) is chosen in the set of alternatives.

Step 4: end.

\section{MDM Example and Comparison with Existing MDM Approaches}

6.1. MDM Example of Software Selection. This section indicates an MDM example of software selection adapted from [9] to reveal the usability and efficiency of the established MDM approach under the environment of TrNZNs.

In an MDM example, an investment company needs to select a suitable software system from potential software systems, where five candidate software systems are provided preliminarily and denoted as a set of five alternatives $Q=$ $\left\{Q_{1}, Q_{2}, Q_{3}, Q_{4}, Q_{5}\right\}$. Then, these alternatives must satisfy the requirements of the four criteria: $s_{1}$ (the contribution to organization performance), $s_{2}$ (the effort to transform from current system), $s_{3}$ (the costs of hardware/software investment), and $s_{4}$ (the outsourcing software developer reliability). Regarding the importance of the four criteria, the weight values of the four criteria are specified as the weight vector $\lambda=(0.25,0.25,0.3,0.2)$. Thus, decision makers/experts assess the satisfiability of the five alternatives over the four criteria by TrNZNs $\widetilde{z}_{i j}=<\left(\left(T_{V i j 1}, T_{V i j 2}, T_{V i j 3}, T_{V i j 4}\right)\right.$, $\left.\left(T_{R i j 1}, T_{R i j 2}, T_{R i j 3}, T_{R i j 4}\right)\right),\left(\left(I_{V i j 1}, I_{V i j 2}, I_{V i j 3}, I_{V i j 4}\right),\left(I_{R i j 1}, I_{R i j 2}\right.\right.$, $\left.\left.I_{R i j 3}, I_{R i j 4}\right)\right),\left(\left(F_{V i j 1}, F_{V i j 2}, F_{V i j 3}, F_{V i j 4}\right),\left(F_{R i j 1}, F_{R i j 2}, F_{R i j 3}, F_{R i j 4}\right)\right)$ $>(j=1,2,3,4 ; i=1,2,3,4,5)$, where $\left(T_{V i j 1}, T_{V i j 2}, T_{V i j 3}, T_{V i j 4}\right)$ $\subseteq[0,1]$ and $\left(T_{R i j 1}, T_{R i j 2}, T_{R i j 3}, T_{R i j 4}\right) \subseteq[0,1]$ indicate that the alternative $Q_{i}$ satisfies the degrees and reliability measures of the criteria $s_{j}$, $\left(I_{V i j 1}, I_{V i j 2}, I_{V i j 3}, I_{V i j 4}\right) \subseteq[0,1]$ and $\left(I_{R i j 1}, I_{R i j 2}\right.$, $\left.I_{R i j 3}, I_{R i j 4}\right) \subseteq[0,1]$ indicate the indeterminate degrees and reliability measures of the alternative $Q_{i}$ over the criteria $s_{j}$, and $\left(F_{V i j 1}, F_{V i j 2}, F_{V i j 3}, F_{V i j 4}\right) \subseteq[0,1]$ and $\left(F_{R i j 1}, F_{R i j 2}, F_{R i j 3}\right.$, $\left.F_{R i j 4}\right) \subseteq[0,1]$ indicate that the alternative $A_{i}$ does not satisfy the degrees and reliability measures of the criteria $s_{j}$, along with $0 \leq T_{V i j 4}+I_{V i j 4}+F_{V i j 4} \leq 3$ and $0 \leq T_{R i j 4}+I_{R i j 4}+F_{R i j 4} \leq 3$. Hence, all the specified TrNZNs can be constructed as the following decision matrix $\widetilde{Z}=\left(\widetilde{z}_{i j}\right)_{5 \times 4}$ : 


$$
\begin{aligned}
& \widetilde{Z}=\left[\begin{array}{l}
\langle((0.4,0.5,0.6,0.7),(0.4,0.5,0.6,0.7)),((0.0,0.1,0.2,0.3),(0.3,0.4,0.5,0.6)),((0.1,0.1,0.1,0.1),(0.3,0.4,0.5,0.6)\rangle \\
\langle((0.3,0.4,0.5,0.5),(0.5,0.6,0.7,0.8)),((0.1,0.2,0.3,0.4),(0.4,0.5,0.6,0.7)),((0.0,0.1,0.1,0.1),(0.5,0.6,0.7,0.8))\rangle \\
\langle((0.1,0.1,0.1,0.1),(0.5,0.6,0.7,0.8)),((0.1,0.1,0.1,0.1),(0.6,0.7,0.8,0.9)),((0.6,0.7,0.8,0.9),(0.5,0.6,0.7,0.8))\rangle \\
\langle((0.7,0.7,0.7,0.7),(0.4,0.5,0.6,0.7)),((0.0,0.1,0.2,0.3),(0.4,0.5,0.6,0.7)),((0.1,0.1,0.1,0.1),(0.3,0.4,0.5,0.6))\rangle \\
\langle((0.0,0.1,0.2,0.2),(0.4,0.5,0.6,0.7)),((0.1,0.1,0.1,0.1),(0.3,0.4,0.5,0.6)),((0.5,0.6,0.7,0.8),(0.5,0.6,0.7,0.8))\rangle
\end{array}\right. \\
& \langle((0.0,0.1,0.2,0.3),(0.3,0.4,0.5,0.6)),((0.0,0.1,0.2,0.3),(0.4,0.5,0.6,0.7)),(0.2,0.3,0.4,0.5),(0.3,0.4,0.5,0.6)\rangle \\
& \langle((0.2,0.3,0.4,0.5),(0.6,0.7,0.8,0.9)),((0.0,0.1,0.2,0.3),(0.5,0.6,0.7,0.8)),((0.0,0.1,0.2,0.3),(0.4,0.5,0.6,0.7))\rangle \\
& \langle((0.0,0.1,0.1,0.2),(0.5,0.6,0.7,0.8)),((0.0,0.1,0.2,0.3),(0.5,0.6,0.6,0.7)),((0.3,0.4,0.5,0.6),(0.3,0.4,0.5,0.6))\rangle \\
& \langle((0.4,0.5,0.6,0.7),(0.5,0.6,0.7,0.8)),((0.1,0.1,0.1,0.1),(0.6,0.7,0.7,0.8)),((0.0,0.1,0.2,0.2),(0.5,0.6,0.7,0.8))\rangle \\
& \langle((0.4,0.4,0.4,0.4),(0.3,0.4,0.5,0.6)),((0.0,0.1,0.2,0.3),(0.4,0.5,0.6,0.7)),((0.0,0.1,0.2,0.3),(0.5,0.6,0.7,0.8))\rangle \\
& \langle((0.3,0.4,0.5,0.6),(0.4,0.5,0.6,0.7)),((0.0,0.1,0.2,0.3),(0.4,0.5,0.5,0.6)),((0.1,0.1,0.1,0.1),(0.4,0.5,0.5,0.6))\rangle \\
& \langle((0.0,0.1,0.1,0.2),(0.5,0.6,0.7,0.8)),((0.1,0.1,0.1,0.1),(0.4,0.5,0.6,0.7)),((0.5,0.6,0.7,0.8),(0.3,0.4,0.5,0.6))\rangle \\
& \langle((0.2,0.3,0.4,0.5),(0.3,0.4,0.5,0.6)),((0.0,0.1,0.2,0.3),(0.5,0.6,0.7,0.8)),((0.1,0.2,0.2,0.3),(0.4,0.5,0.6,0.7))\rangle \\
& \langle((0.2,0.3,0.4,0.5),(0.5,0.6,0.6,0.7)),((0.0,0.1,0.2,0.3),(0.5,0.6,0.7,0.8)),((0.1,0.2,0.3,0.3),(0.3,0.4,0.5,0.6))\rangle \\
& \langle((0.6,0.7,0.7,0.8),(0.5,0.5,0.5,0.5)),((0.1,0.1,0.1,0.1),(0.5,0.6,0.7,0.8)),((0.0,0.1,0.1,0.2),(0.4,0.5,0.5,0.6))\rangle \\
& \langle((0.3,0.4,0.5,0.6),(0.3,0.4,0.5,0.6)),((0.1,0.1,0.1,0.1),(0.4,0.5,0.6,0.7)),((0.1,0.2,0.3,0.4),(0.5,0.6,0.6,0.7))\rangle] \\
& \langle((0.3,0.4,0.5,0.5),(0.5,0.6,0.6,0.7)),((0.0,0.1,0.2,0.3),(0.3,0.4,0.5,0.6)),((0.0,0.1,0.1,0.2),(0.4,0.5,0.6,0.7))\rangle \\
& \langle((0.1,0.2,0.3,0.4),(0.6,0.7,0.8,0.9)),((0.1,0.1,0.1,0.1),(0.5,0.6,0.7,0.8)),((0.3,0.4,0.5,0.6),(0.4,0.5,0.6,0.7))\rangle \\
& \langle((0.1,0.2,0.3,0.4),(0.5,0.6,0.7,0.8)),((0.1,0.1,0.1,0.1),(0.4,0.5,0.5,0.6)),((0.4,0.5,0.6,0.6),(0.3,0.4,0.5,0.6))\rangle \\
& \langle((0.1,0.2,0.3,0.3),(0.4,0.5,0.6,0.7)),((0.1,0.2,0.3,0.4),(0.4,0.5,0.5,0.6)),((0.2,0.3,0.4,0.5),(0.4,0.5,0.6,0.7))\rangle]
\end{aligned}
$$

Thus, we utilize the established MDM approach to obtain the most suitable software system(s), which can be depicted by the following decision process.

First, by equation (19) or equation (20), we obtain the following aggregated TrNZNs $\widetilde{z}_{i}(i=1,2,3,4,5)$ :

$\widetilde{z}_{1}=<((0.2636,0.3656,0.4682,0.5719),(0.3569,0.4572$, $0.5577,0.6585)),((0,0.1000,0.1741,0.2408),(0.3722$, $0.4729, \quad 0.5428,0.6431)), \quad((0.1189,0.1512,0.1762$, $0.1973),(0.3622,0.4638,0.5186,0.6188))>$

$\widetilde{z}_{2}=<((0.1945,0.2958,0.3758,0.4243),(0.5271,0.6278$, $0.7129,0.8176)),((0,0.1189,0.1798,0.2319),(0.3993$, $0.5005,0.6012,0.7018)),((0,0.1712,0.2132,0.2821)$, $(0.3880,0.4894,0.5904,0.6911))>$

$\widetilde{z}_{3}=<((0.1081,0.1848,0.2421,0.3245),(0.4710,0.5735$, $0.6776,0.7856)),((0,0.1000,0.1464,0.1830),(0.5233$, $0.6236,0.6964,0.7969)), \quad((0.2566,0.3737,0.4272$, 0.5393), (0.3936, 0.4949, 0.5958, 0.6964))>

$\widetilde{z}_{4}=<((0.4035,0.4652,0.5298,0.5983),(0.4767,0.5771$, $0.6486,0.7500)),((0,0.1000,0.1464,0.1830),(0.4733$, $0.5745,0.6297,0.7305)),((0,0.1699,0.2366,0.2366)$, $(0.3409,0.4427,0.5439,0.6447))>$

$\widetilde{z}_{5}=<((0.3454,0.4287,0.4599,0.5218),(0.4096,0.4767$, $0.5478,0.6242)),((0,0.1149,0.1481,0.1737),(0.3980$, $0.4995,0.5789,0.6798)),((0,0.1950,0.2552,0.3760)$, $(0.4472,0.5477,0.6136,0.7145))>$

Or we obtain the following aggregated $\operatorname{TrNZNs} \widetilde{z}_{i}(i=1$, $2,3,4,5)$ :

$\widetilde{z}_{1}=<((0,0.2991,0.4162,0.5244), \quad(0.3514,0.4522$, $0.5527,0.6531)),((0.0209,0.1000,0.1809,0.2639)$, $(0.3764,0.4767,0.5478,0.6486)),((0.1261,0.1745$, $0.2266,0.2835),(0.3751,0.4762,0.5218,0.6224))>$ $\widetilde{z}_{2}=<((0,0.2456,0.2918,0.3798),(0.5233,0.6236$, $0.7018,0.8022)),((0.0563,0.1261,0.1984,0.2737)$, $(0.4088,0.5096,0.6108,0.7129)), \quad((0.1877,0.2944$, $0.3715,0.4743),(0.3996,0.5005,0.6020,0.7045))>$

$\widetilde{z}_{3}=<((0,0.1597,0.1888,0.2543),(0.4449,0.5479$, $0.6499,0.7513)),((0.0463,0.1000,0.1565,0.2162)$, $(0.5271,0.6278,0.7087,0.8139)),((0.3437,0.4500$, $0.5422,0.6655),(0.4042,0.5051,0.6064,0.7087))>$

$\widetilde{z}_{4}=<((0.2832,0.3885,0.4807,0.5658),(0.4729,0.5733$, $0.6431,0.7434)),((0.0463,0.1000,0.1565,0.2162)$, $(0.4867,0.5884,0.6430,0.7458)),((0.1480,0.2276$, $0.3109,0.3109),(0.3565,0.4578,0.5599,0.6636))>$

$\widetilde{z}_{5}=<((0,0.2912,0.3756,0.3910), \quad(0.3980,0.4729$, $0.5428,0.6089)),((0.0760,0.1210,0.1690,0.2206)$, $(0.4096,0.5106,0.5943,0.6976)),((0.1958,0.3012$, $0.3877,0.5020),(0.4523,0.5528,0.6296,0.7330))>$

Then, the results of the MDM approach based on the TrNZNWAA and TrNNWGA operators and the score function are shown in Table 1.

From the results of Table 1, the ranking orders based on the TrNZNWAA and TrNZNWGA operators are identical and the best one indicates the same selection as the software system $Q_{4}$.

6.2. Comparison with Existing MDM Approaches. For convenient comparison with existing MDM approach in the setting of TrNNs [9], we may ignore the reliability measures in TrNZNs and only contain the decision matrix of TrNNs in the MDM example as its special case. Thus, existing MDM approach in the setting of TrNNs [9] can be used for the special case of the MDM example. In this case, the decision results based on the TrNNWAA and TrNNWGA operators 
TABLE 1: Results of the MDM approach based on the TrNZNWAA and TrNZNWGA operators and the score function.

\begin{tabular}{lcc}
\hline $\begin{array}{l}\text { Aggregation } \\
\text { operator }\end{array}$ & Score value & Ranking \\
TrNZNWAA & $0.6892,0.6845,0.6154$, & Q4 $>$ Q1 $>$ Q2 $>$ \\
& $0.7207,0.6824$ & Q5 $>$ Q3 \\
TrNZNWGA & $0.6607,0.6257,0.5750$, & Q4 $>$ Q1 $>$ Q2 > \\
& $0.6848,0.6158$ & Q5 $>$ Q3 \\
\hline
\end{tabular}

TABLE 2: Results of the MDM approach based on the TrNNWAA and TrNNWGA operators and the score function [9].

\begin{tabular}{lcc}
\hline $\begin{array}{l}\text { Aggregation } \\
\text { operator }\end{array}$ & Score value & Ranking \\
TrNNWAA & $0.7092,0.6744,0.5694$, & Q4 $>$ Q1 $>$ Q5 $>$ \\
& $0.7437,0.7077$ & Q2 $>$ Q3 \\
TrNNWGA & $0.6553,0.5779,0.5069$, & Q4 $>$ Q1 $>$ Q5 $>$ \\
& $0.6835,0.5904$ & Q2 $>$ Q3 \\
\hline
\end{tabular}

(equations (2) and (3)) and the score function of TrNNs (equation (4)) are introduced from [9], which are shown in Table 2.

Based on the decision results in Tables 1 and 2, we can see that the ranking orders based on the established MDM approach and the existing MDM approach [9] reveal their difference, but the best alternative $Q_{4}$ (the best software system) is identical. Then, the reason for their ranking difference is that decision information in the existing MDM approach [9] only contains $\mathrm{TrNN}$ s without considering the reliability measures of TrNNs in this MDM example, while decision information in the established MDM approach contains both TrNNs and their reliability measures. Hence, different decision information can result in different ranking results. It is obvious that the reliability measures in this example can affect the ranking order of alternatives, which shows the efficiency and rationality of the established MDM approach under the environment of TrNZNs.

However, the different decision information and decision methods can have an impact on the ranking of alternatives in the MDM problem, which reveals their importance in MDM applications. Thus, existing MDM methods [11-14, 23] only contain the TrNN or NZN information without considering the reliability measures in TrNNs or continuous Z-numbers in NZNs; they may lose some useful decision information so as to result in decision distortion/unreasonable decision results, which reveal some insufficiencies, while the new established approach can contain much more information than existing MDM methods and overcome the insufficiencies. Furthermore, existing methods $[11-14,23]$ also cannot deal with such MDM problems with TrNZNs.

Based on the above comparative analysis, the new established approach in setting of TrNZNs not only makes assessment information of TrNNs more reliable but also strengthens the effectiveness and continuity of decision information by comparison with existing MDM methods with TrNN and NZN information [9, 11-14, 23], which reveals the highlighting advantages of the new established approach in the information representation and MDM applications. Therefore, the new established approach not only extends existing methods but also demonstrates its superiority over them.

\section{Conclusion}

To make $\operatorname{TrNN}$ reliable, this paper presented a $\operatorname{TrNZN}$ set based on the truth, falsity, and indeterminacy trapezoidal $\mathrm{Z}$-numbers as the generalization of the Z-number concept and then defined basic operations of TrNZNs, score and accuracy functions of TrNZNs, and ranking laws of TrNZNs. Next, the TrNZNWAA and TrNZNWGA operators were proposed to aggregate the TrNZN information. Furthermore, an MDM approach based on the two aggregation operators and score and accuracy functions was established in the setting of TrNZNs, in which the assessment values of alternatives over the criteria take the form of TrNZNs containing TrNNs and their reliability measures. Finally, an MDM example of software selection was provided to reveal the suitability and efficiency of the established MDM approach in the setting of TrNZNs.

The main advantage of this study is that the established method not only makes assessment information of TrNNs more reliable but also strengthens the decision rationality and efficiency in solving MDM problems with TrNZN information. However, the established method only uses the basic aggregation algorithms of TrNZNWAA and TrNZNWGA for MDM problems without considering the interactions of some evaluation criteria with each other, which implies the limitation of the proposed method in MDM applications. For capturing these relationships, the future study is to develop other aggregation algorithms and to use them for some other MDM problems including slope design schemes, energy and environmental managements, and medicine options.

\section{Data Availability}

There are no underlying data supporting the results of your study.

\section{Conflicts of Interest}

The authors declare no conflicts of interest.

\section{References}

[1] M. Akram, S. Naz, and F. Smarandache, "Generalization of maximizing deviation and TOPSIS method for MADM in simplified neutrosophic hesitant fuzzy environment," Symmetry, vol. 11, no. 8, p. 1058, 2019.

[2] J. Zhan, M. Akram, and M. Sitara, "Novel decision-making method based on bipolar neutrosophic information," Soft Computing, vol. 23, no. 20, pp. 9955-9977, 2019.

[3] S. Habib, W. us Salam, M. A. Butt, M. Akram, and F. Smarandache, "A neutrosophic clinical decision-making system for cardiovascular diseases risk analysis," Journal of Intelligent \& Fuzzy Systems, vol. 39, no. 5, pp. 7807-7829, 2020.

[4] F. Liu and X. H. Yuan, "Fuzzy number intuitionistic fuzzy set," Fuzzy Systems and Mathematics, vol. 21, no. 1, pp. 88-91, 2007. 
[5] X. F. Wang, "Fuzzy number intuitionistic fuzzy geometric aggregation operators and their application to decision making," Control and Decision, vol. 23, no. 6, pp. 607-612, 2008.

[6] X. F. Wang, "Fuzzy number intuitionistic fuzzy arithmetic aggregation operators," International Journal of Fuzzy Systems, vol. 10, no. 2, pp. 104-111, 2008.

[7] G. Wei, X. Zhao, and R. Lin, "Some induced aggregating operators with fuzzy intuitionistic number information and their applications for group decision making," International Journal of Computational Intelligence Systems, vol. 3, no. 1, pp. 84-95, 2010.

[8] J. Ye, "Prioritized aggregation operators of trapezoidal intuitionistic fuzzy sets and their application to multicriteria decision-making," Neural Computing and Applications, vol. 25, no. 6, pp. 1447-1454, 2014.

[9] J. Ye, "Trapezoidal neutrosophic set and its application to multiple attribute decision-making," Neural Computing and Applications, vol. 26, no. 5, pp. 1157-1166, 2015.

[10] R. Liang, J. Wang, and H. Zhang, "Evaluation of e-commerce websites: an integrated approach under a single-valued trapezoidal neutrosophic environment," Knowledge-Based Systems, vol. 135, pp. 44-59, 2017.

[11] İ. Deli, "A novel defuzzification method of SV-trapezoidal neutrosophic numbers and multi-attribute decision making: a comparative analysis," Soft Computing, vol. 23, no. 23, pp. 12529-12545, 2019.

[12] B. C. Giri, M. U. Molla, and P. Biswas, "TOPSIS method for MADM based on interval trapezoidal neutrosophic number," Neutrosophic Sets and Systems, vol. 22, pp. 151-167, 2018.

[13] C. Jana, M. Pal, F. Karaaslan, and J. Wang, "Trapezoidal neutrosophic aggregation operators and their application to the multi-attribute decision-making process," Scientia Iranica, vol. 27, no. 3, pp. 1655-1673, 2020.

[14] K. Khatter, "Interval valued trapezoidal neutrosophic set: multi-attribute decision making for prioritization of nonfunctional requirements," Journal of Ambient Intelligence and Humanized Computing, vol. 12, pp. 1039-1055, 2020.

[15] L. A. Zadeh, "A note on z-numbers," Information Sciences, vol. 181, no. 14, pp. 2923-2932, 2011.

[16] P. Patel, E. S. Khorasani, and S. Rahimi, "Modeling and implementation of z-number," Soft Computing, vol. 20, no. 4, pp. 1341-1364, 2016.

[17] B. Kang, Y. Hu, Y. Deng, and D. Zhou, "A new methodology of multicriteria decision-making in supplier selection based onZ-numbers," Mathematical Problems in Engineering, vol. 2016, Article ID 8475987, 17 pages, 2016.

[18] W. Jiang, C. Xie, M. Zhuang, Y. Shou, and Y. Tang, "Sensor data fusion with z-numbers and its application in fault diagnosis," Sensors, vol. 16, no. 9, pp. 1-22, 2016.

[19] R. A. Aliev, W. Pedrycz, O. H. Huseynov, and S. Z. Eyupoglu, "Approximate reasoning on a basis of z-number-valued ifthen rules," IEEE Transactions on Fuzzy Systems, vol. 25, no. 6, pp. 1589-1600, 2017.

[20] D. Qiu, R. Dong, S. Chen, and A. Li, "On an optimization method based on Z-numbers and the multi-objective evolutionary algorithm," Intelligent Automation and Soft Computing, vol. 24, no. 1, pp. 147-150, 2018.

[21] B. Kang, P. Zhang, Z. Gao, G. Chhipi-Shrestha, K. Hewage, and R. Sadiq, "Environmental assessment under uncertainty using Dempster-Shafer theory and Z-numbers," Journal of Ambient Intelligence and Humanized Computing, vol. 11, no. 5, pp. 2041-2060, 2020.
[22] Z. Ren, H. Liao, and Y. Liu, "Generalized Z-numbers with hesitant fuzzy linguistic information and its application to medicine selection for the patients with mild symptoms of the COVID-19," Computers \& Industrial Engineering, vol. 145, 2020 UNSP 106517, Article ID 106517.

[23] S. G. Du, J. Ye, R. Yong, and F. W. Zhang, "Some aggregation operators of neutrosophic Z-numbers and their multicriteria decision making method," Complex \& Intelligent Systems, vol. 7, pp. 429-438, 2021. 